\title{
Starburst in HS $0822+3542$ induced by the very blue LSB dwarf SAO 0822+3545
}

\author{
S. A. Pustilnik ${ }^{1,4}$, A. Y. Kniazev ${ }^{1,2,4}$, A. G. Pramskij ${ }^{1,4}$, A. V. Ugryumov ${ }^{1,4}$, and J. Masegosa ${ }^{3}$ \\ 1 Special Astrophysical Observatory RAS, Nizhnij Arkhyz, Karachai-Circassia 369167, Russia \\ 2 Max Planck Institut für Astronomie, Königstuhl 17, 69117 Heidelberg, Germany \\ 3 Instituto de Astrofisica de Andalucía, Granada, Spain \\ ${ }^{4}$ Isaac Newton Institute of Chile, SAO Branch
}

Received 26 February 2002 / Accepted 30 July 2003

\begin{abstract}
One of the most metal-deficient blue compact galaxies (BCGs) HS $0822+3542\left(Z=1 / 34 Z_{\odot}\right)$, is also one of the nearest such objects $(D \sim 11 \mathrm{Mpc})$. It is in addition well isolated from known bright galaxies. A trigger mechanism for its current star-formation (SF) burst has thus remained unclear. We report the discovery of a very blue $\left((B-V)_{\mathrm{tot}}^{0}=0.08\right.$ and $\left.(V-R)_{\text {tot }}^{0}=0.14\right)$ low surface brightness (LSB) $\left(\mu_{B}^{0} \gtrsim 23.4 \operatorname{arcsec}^{-2}\right)$ dwarf irregular (dIrr) galaxy, which we have named SAO $0822+3545$. Its small relative velocity and projected distance of only $\sim 11 \mathrm{kpc}$ from the BCG imply their physical association. For this LSB dIrr galaxy, we present spectroscopic results, total $B, V, R$ magnitudes, and the effective radii and surface brightness (SB), and we describe its morphological properties. We compare the very blue colours of this dwarf with PEGASE. 2 models of the colour evolution of a $Z=1 / 20 Z_{\odot}$ stellar population, and combine this analysis with the data on the $\operatorname{LSBD} E W(\mathrm{H} \alpha)$ values. The models best describing all available observational data depend on the relative fraction of massive stars in the IMF used. For a Salpeter IMF with $M_{\text {up }}=120 M_{\odot}$, the best model includes a "young" single stellar population (SSP) with an age of $\sim 10 \mathrm{Myr}$ and an "old" SSP with the age of $\sim 0.2-10 \mathrm{Gyr}$. The mass ratio of the old to young components should be in the range of 10 to 30 . If the age of the old component is more than $\sim 1 \mathrm{Gyr}$, an additional coeval component of "intermediate" age ( 100 Myr) with a mass comparable to that of the "young" population, although not required, provided a good fit to the current data. For the two options of a model IMF biased toward the low-mass end, the best match of the observed $B V R$ and $\mathrm{EW}(\mathrm{H} \alpha)$ is for continuous star-formation rate (SFR) single-component models, with SF durations in the range of $\sim 0.1$ to $\sim 1$ Gyr. However, only a longer time-scale SF gives the stellar mass, compatible with the LSB galaxy mass estimates. Nevertheless, such a scenario would be inconsistent with the recent encounter of these two dwarfs. The role of interaction between the LSBD and BCG HS $0822+3542$ in triggering their major SF episodes during the last $~ 100-200 \mathrm{Myr}$ is emphasized and discussed. For the BCG, based on the results of new spectroscopy with the Russian $6 \mathrm{~m}$ telescope, we estimate the physical parameters of its SF region and present the first evidence of an ionized gas supershell. This pair of dwarfs lies deep within the nearby Lynx-Cancer void, with the nearest bright $\left(L>L_{*}\right)$ galaxies at distances $>3 \mathrm{Mpc}$. This is probably one of the main factors responsible for the unevolved state of HS $0822+3542$.
\end{abstract}

Key words. galaxies: interactions - galaxies: photometry - galaxies: abundances - galaxies: individual: HS 0822+3542, SAO $0822+3545$

\section{Introduction}

A few known blue compact galaxies (BCGs) with extremely low metallicities $\left(1 / 50\right.$ to $\left.1 / 20 Z_{\odot}\right)$ are considered to be the best candidates for truly young local low-mass galaxies, in which we are witnessing the first star formation episode, with the oldest stars formed less than $\sim 100-200 \mathrm{Myr}$ ago. The best known examples are SBS 0335-052 (Izotov et al. 1997; Papaderos et al. 1998; Pustilnik et al. 2001a), and I Zw 18 (Searle \& Sargent 1972; Izotov \& Thuan 1999;

Send offprint requests to: S. Pustilnik, e-mail: sap@sao.ru
Papaderos et al. 2002) ${ }^{1}$. A question then arises: why did their progenitors - protogalactic H I clouds not experience gas collapse earlier, during the time after their neutral gas settled down into the gravitational wells of their dark matter halos? Recent observational data indicate that some of them certainly interact with their nearest neighbours (e.g., Dw 1225+0152, Chengalur et al. 1995; SBS 0335-052 E, Pustilnik et al. 2001a; and probably IZw18 with the tiny object IZw18C, Dufour et al. 1996;

\footnotetext{
${ }^{1}$ Note that debates on the possible youth of these BCGs still continue in the literature (see, e.g., the most recent Östlin 2000; Östlin \& Kunth 2001; Kunth \& Östlin 2001).
} 
van Zee et al. 1998; Izotov et al. 2001). This, presumably, is a key moment of their history. Moreover, it is probably not by chance that in both the cases of Dw 1225+0152 and SBS 0335-052 E the nearest neighbour is very gas-rich, and either is an $\mathrm{H}$ I cloud without any hint of past star formation (as in the case of Dw 1225+0152, Salzer et al. 1991), or is also an extremely metal-deficient, probably truly young, blue compact galaxy (SBS 0335-052 W, Pustilnik et al. 1997; Lipovetsky et al. 1999).

Low surface brightness galaxies (LSBGs) comprise a large fraction of the general field galaxy population and outnumber by a factor of several times the high surface brightness (HSB) population (e.g., McGaugh 1996; Dalcanton et al. 1997; O'Neil \& Bothun 2000). Therefore, these LSBGs can be an important factor for interaction-induced star-formation activity in gas-rich galaxies in general, mostly through distant/weak tidal encounters (see, e.g., Taylor et al. 1995; O’Neil et al. 1998; Pustilnik et al. 2001b). In particular, through interaction they can trigger the first starbursts in the hypothetical population of local protogalactic H I clouds. To check this hypothesis the authors are conducting a systematic study of the local environment of the most metal-deficient BCGs. We present here new evidence in support of this idea. We report the discovery of a LSB dwarf irregular galaxy (named SAO 0822+3545) at a projected distance of $\sim 11.4 \mathrm{kpc}$ from one of the most metaldeficient BCGs, HS 0822+3542 (Kniazev et al. 2000). We have used the SAO RAS $6 \mathrm{~m}$ telescope spectrum in the $\mathrm{H} \alpha$ region, as well as $B V R$ photometry from the Nordic Optical Telescope (NOT) to study its properties and estimate its tidal effect on the BCG HS 0822+3542. We also present new high signal-to-noise (S/N) $6 \mathrm{~m}$ telescope spectra for HS $0822+3542$ which allow us to make more accurate measurements of some of the physical parameters of its star-forming (SF) region and discover the first kinematic evidence of an ionized gas supershell. In Sect. 2 we describe the observations and their reduction. Results of the data analysis are presented in Sect. 3. We discuss these results in Sect. 4, and summarize our findings and draw conclusions in Sect. 5 .

\section{Observations and reduction}

\subsection{Nordic Optical Telescope photometry}

There are no cataloged galaxies around HS $0822+3542$ in either NED or LEDA databases with $V_{\text {hel }}<1200 \mathrm{~km} \mathrm{~s}^{-1}$ and projected distances less than $5.8^{\circ}$. To search for fainter/noncataloged galaxies in the close vicinity of this BCG we used $B, V, R$ CCD images obtained with the $2.5 \mathrm{~m}$ NOT on May 28, 1998. These are the same frames which were used to derive $B, V, R$ magnitudes for HS $0822+3542$. They were acquired with the ALFOSC spectrograph equipped with the LORAL (W11-3AC) CCD, which has a direct imaging mode. Exposure times were $900 \mathrm{~s}$ for the $B$-band image, and $600 \mathrm{~s}$ for both $V$ and $R$ images. For further details of these observations and data reduction we refer to the paper by Kniazev et al. (2000).

\subsection{Looking for possible companions}

The photometric data were reduced with MIDAS ${ }^{2}$ Command Language programs according to the method described in Kniazev (1997). The MIDAS INVENTORY package was used to classify all objects. Isophotal and total $B V R$ magnitudes were computed using the transformation coefficients of Kniazev et al. (2000).

Since the interaction between HS $0822+3542$ and a possible neighbouring galaxy would probably result in some enhanced star formation in the latter, we first of all searched for candidate blue galaxies. The second criterion applied was that the brightness of any candidates should be comparable to that of HS $0822+3542$, since significantly fainter neighbours could tidally affect the BCG only from very small distances (see, e.g., estimates in Pustilnik et al. 2001b).

Only two blue galaxies were found in the examined field. The first one is HS $0822+3542$ itself. The second is a LSB irregular galaxy (SAO $0822+3545)$ at $3.5^{\prime}$ to the north-east from HS $0822+3542$ (its coordinates are given in Table 5). The Digitized Sky Survey (DSS-II) blue image of this field with the 2 objects of interest and Anon J0825+3534 is presented in Fig. 1. All other galaxies in the NOT field were either significantly fainter or redder.

\subsection{Analysis of photometric data}

We performed the reduction of photometric data for SAO $0822+3545$ using the IRAF $^{3}$ package ELLIPSE. The growth curve (GC) of the galaxy was constructed by summing up the pixel values from the center outwards in the circles of successive radius.

The total magnitudes $\left(B_{\text {tot }}, V_{\text {tot }}, R_{\text {tot }}\right.$ ) were estimated by asymptotic extrapolation of the respective radial GCs. Modelindependent parameters were derived from each growth curve. The effective radii $\left(r_{\text {eff }}\right)$ were read on each GC at one half the asymptotic intensity, and effective SBs ( $\left.S B_{\text {eff }}\right)$ were determined as the mean brightness within a circle with the effective radius. These values are summarized in Table 2.

\subsection{Long-slit spectroscopy}

Spectroscopic data were obtained with the $6 \mathrm{~m}$ telescope of the Special Astrophysical Observatory of Russian Academy of Sciences (SAO RAS). The long-slit spectrograph (LSS) (Afanasiev et al. 1995) was used with a Photometrics $1024 \times 1024$ pixel CCD detector with a $24 \mu \mathrm{m}$ pixel size. The wavelength ranges of the spectra obtained for different hardware configurations are given in Table 1. Reference spectra of an $\mathrm{Ar}-\mathrm{Ne}-\mathrm{He}$ lamp were recorded before or after each observation to provide wavelength calibration. All observations were

\footnotetext{
${ }^{2}$ MIDAS is an acronym for the European Southern Observatory package - Munich Image Data Analysis System.

3 IRAF: the Image Reduction and Analysis Facility is distributed by the National Optical Astronomy Observatories, which is operated by the Association of Universities for Research in Astronomy, In. (AURA) under cooperative agreement with the National Science Foundation (NSF).
} 


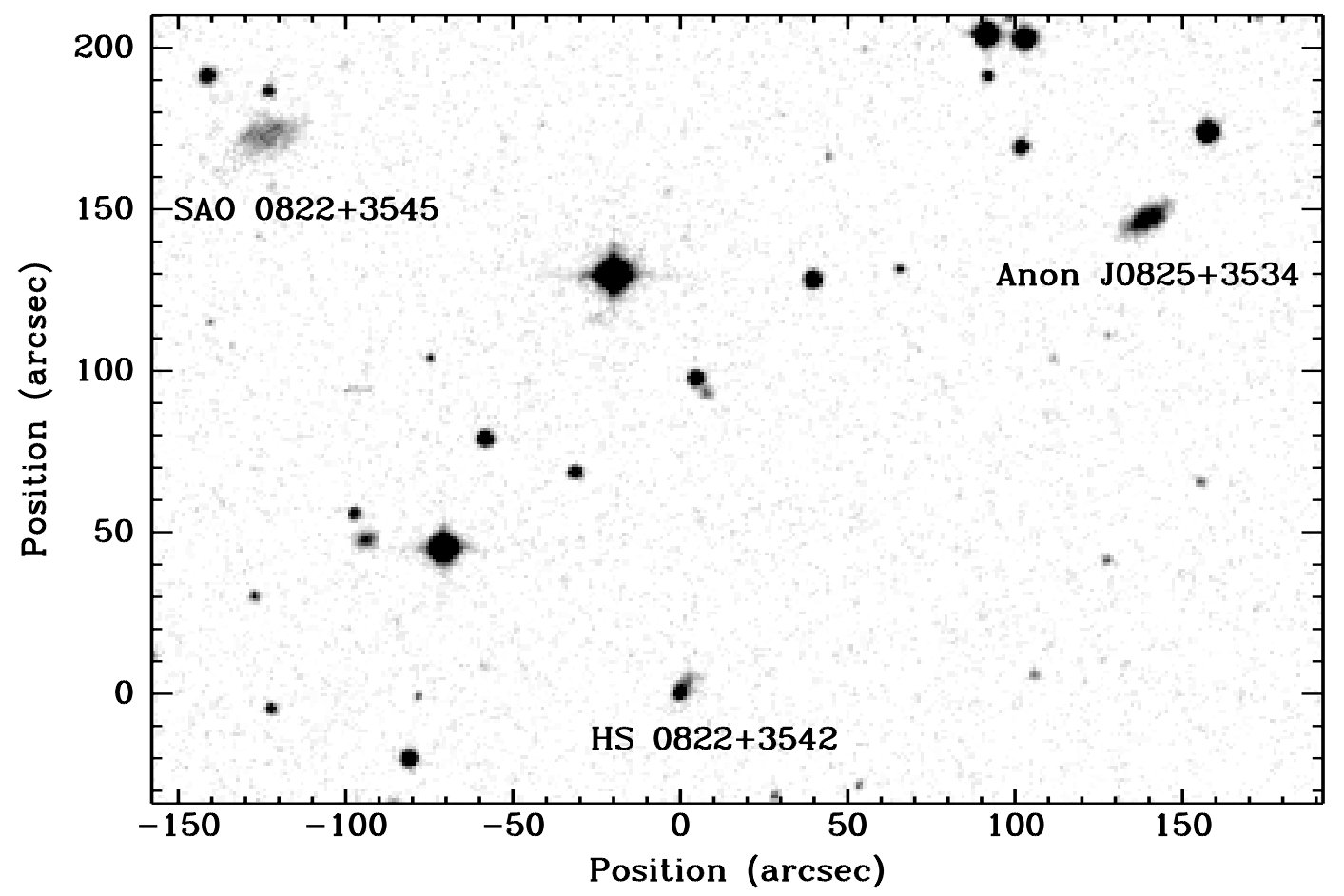

Fig. 1. The Digitized Sky Survey (DSS-II) blue image of the field in the vicinity of BCG HS $0822+3542$. North is up, East is to the left. At the adopted distance of HS $0822+3542(11 \mathrm{Mpc})$ the scale is $\sim 54 \mathrm{pc}=1^{\prime \prime}$. The two marked galaxies - LSBD SAO $0822+3545$ and Anon J0825+3534 have been checked as possible companions of the BCG. On our data the LSBD has $V_{\text {hel }} \sim 700 \mathrm{~km} \mathrm{~s}^{-1}$, close to that of HS $0822+3542$. Its projected distance to the BCG is $213^{\prime \prime}$ or $11.4 \mathrm{kpc}$. The galaxy Anon J0825+3534, very close to the position of $2 \mathrm{MASXi}$ $\mathrm{J} 0825440+353459$ (NED), is a distant background object with $V_{\text {hel }}=15590 \mathrm{~km} \mathrm{~s}^{-1}$.

Table 1. Journal of the $6 \mathrm{~m}$ telescope spectroscopic observations.

\begin{tabular}{|c|c|c|c|c|c|c|c|}
\hline (1) & (2) & $\begin{array}{c}\text { Exposure } \\
\text { time }[\mathrm{s}] \\
\text { (3) }\end{array}$ & 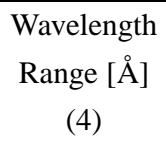 & $\begin{array}{c}\text { Dispersion } \\
{[\AA / \text { pixel }]} \\
\text { (5) }\end{array}$ & $\begin{array}{c}\text { Seeing } \\
\text { [arcsec] } \\
(6)\end{array}$ & (7) & $\begin{array}{c}\text { PA } \\
\text { [degree] } \\
(8)\end{array}$ \\
\hline HS $0822+3542$ & 12.02.1999 & $2 \times 1800$ & $3700-8000$ & 4.6 & 1.8 & 1.10 & 142 \\
\hline HS $0822+3542$ & 13.02.1999 & $2 \times 1800$ & $3700-8000$ & 4.6 & 1.4 & 1.31 & 142 \\
\hline HS $0822+3542$ & 01.11.1999 & $2 \times 1800$ & $6200-7400$ & 1.2 & 1.7 & 1.02 & 175 \\
\hline SAO $0822+3545$ & 17.01.2001 & 1800 & $5700-8100$ & 2.4 & 2.0 & 1.03 & 134 \\
\hline Anon J0825+3534 & 02.02 .2000 & 300 & $5700-8100$ & 2.4 & 2.4 & 1.09 & 121 \\
\hline
\end{tabular}

conducted mainly with the software package NICE in MIDAS, described by Kniazev \& Shergin (1995).

$\mathrm{H} \alpha$ emission from SAO $0822+3545$ was detected in observations on January 17,2001 , with a 651 groove $\mathrm{mm}^{-1}$ grating and a slit width of $2^{\prime \prime}$ (along the major axis), resulting in $2.4 \AA$ pixel $^{-1}$ and a full width at half maximum (FWHM) resolution of $\sim 7 \AA$.

Two high $\mathrm{S} / \mathrm{N}$ spectra of HS $0822+3542$ with the 325 groove $\mathrm{mm}^{-1}$ grating, giving a sampling of $4.6 \AA$ pixel ${ }^{-1}$ and an effective resolution of $\sim 14 \AA(F W H M)$, were obtained during 2 photometric nights on February 12 and 13, 1999. Seeing on these nights was $\sim 1.8^{\prime \prime}$ and $\sim 1.4^{\prime \prime}$, respectively. To keep the long slit $\left(1.2^{\prime \prime} \times 120^{\prime \prime}\right)$ at the same position and PA (position angle), we used the method of differential pointing, similar to that described by Kniazev et al. (2001). Spectrophotometric standard stars from Bohlin (1996) were observed for flux calibration.

The radial velocity distribution of ionized gas along the slit, as seen in the $\mathrm{H} \alpha$-line (Position-Velocity or P-V diagram) was derived for HS $0822+3542$ from the LSS observations on November 1, 1999, with the 1302 groove $\mathrm{mm}^{-1}$ grating, giving a sampling of $1.2 \AA$ pixel $^{-1}$ and an effective resolution (with the $2^{\prime \prime}$ slit width) of $3.5 \AA$. Seeing during these observations was $1.7^{\prime \prime}$.

For the reduction of $2 \mathrm{D}$ spectra we obtained biases, flat fields and illumination correction images. The primary reduction consisted of standard steps and was done using the IRAF package CCDRED. The IRAF package LONGSLIT was used to 


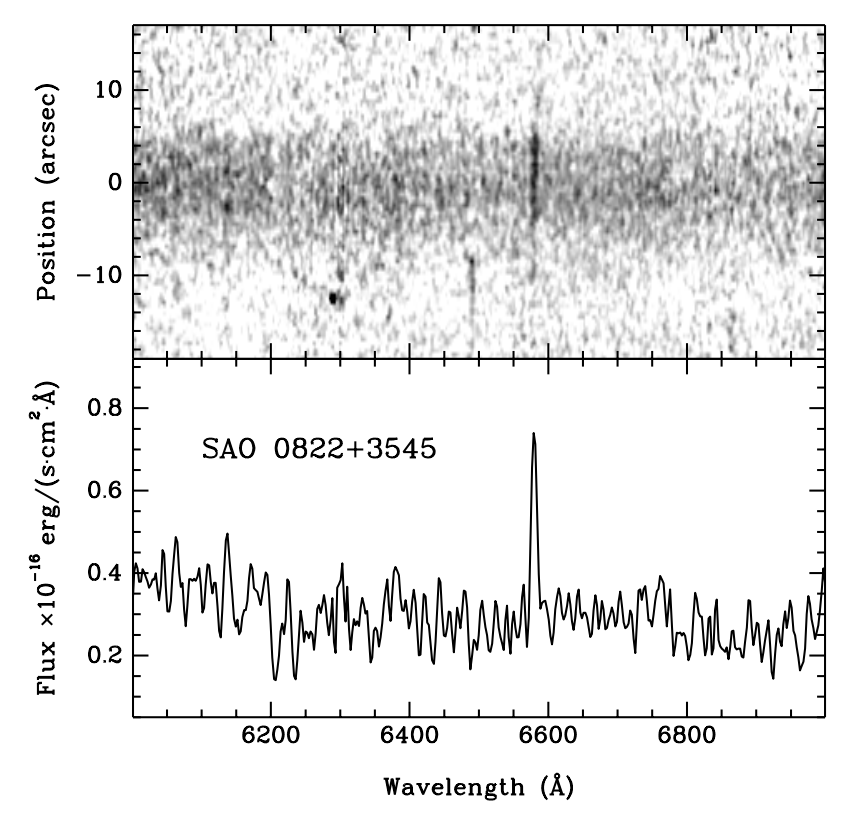

Fig. 2. Top panel: 2D spectrum of SAO $0822+3545$ with the $\mathrm{H} \alpha$ line at $\lambda 6579 \AA$. Positive $Y$ corresponds to the NW direction for the slit position shown in Fig. 3. H $\alpha$-emission is clearly seen on top of the continuum in the region of $\pm \sim 5^{\prime \prime}$ from the central position. Furthermore, some very faint $\mathrm{H} \alpha$-emission (with $\mathrm{S} / \mathrm{N}$ ratio of $\sim 1$ ) can be traced out to $\pm \sim 10^{\prime \prime}$, in the regions without detectable continuum. Bottom panel: 1D spectrum of the central region $\left( \pm 3^{\prime \prime}\right)$, summed along the slit, $E W(\mathrm{H} \alpha)=17 \AA$.

perform wavelength calibration, background subtraction, extinction correction and flux calibration. Straightening of the 2D spectra was performed using APALL, another IRAF package. After that, aperture extractions, continuum determination, flux and equivalent width measurements of spectral lines were performed in MIDAS (for details, see Kniazev et al. 2000). The reduced $2 \mathrm{D}$ spectrum and extracted averaged $1 \mathrm{D}$ spectrum of SAO $0822+3545$ are shown in Fig. 2.

Fluxes and equivalent widths of blended lines were measured using Gaussian decomposition fitting. An average sensitivity curve was produced for each night with rms deviations of $\$ 5 \%$ in the whole spectral range. The sensitivity curve and line intensity errors have been propagated in calculating elemental abundances. To construct the P-V diagram, the methodology described in Zasov et al. (2000) was used, which allowed us to measure points with sufficiently bright $\mathrm{H} \alpha$ emission with rms errors on the level of $2-4 \mathrm{~km} \mathrm{~s}^{-1}$.

\section{Results}

\subsection{Spectrum of SAO $0822+3545$}

The 2D spectrum of SAO $0822+3545$ in Fig. 2 shows $\mathrm{H} \alpha$ emission spanning $\sim 10^{\prime \prime}$, comparable to the apparent extent of the galaxy continuum. Some fainter $\mathrm{H} \alpha$ features can be detected in the peripheral regions, where no continuum is seen in our spectrum. The equivalent width of the $\mathrm{H} \alpha$ line, measured on the $1 \mathrm{D}$ spectrum averaged over the central region with the a spatial extent of $6^{\prime \prime}$, is $E W(\mathrm{H} \alpha)=17 \pm 2 \AA$. The integrated flux in this line is $4.65( \pm 0.55) \times 10^{-16} \mathrm{erg} \mathrm{s}^{-1} \mathrm{~cm}^{-2}$. Small variations of $E W(\mathrm{H} \alpha)$ along the slit near the center of the galaxy on the level of $\sim 15-20 \AA$ are within the observational uncertainties. Closer to the edges the $E W(\mathrm{H} \alpha)$ can be larger, but there the uncertainty reaches $70-100 \%$, so we do not discuss these regions further. The measured velocity $V_{\text {hel }}=700 \pm 50 \mathrm{~km} \mathrm{~s}^{-1}$ is very close to that of HS $0822+3542$.

\subsection{Photometry and morphology of SAO $0822+3545$}

We calculated total magnitudes $B_{\text {tot }}=17.56 \pm 0.05, V_{\text {tot }}=$ $17.43 \pm 0.03$ and $R_{\text {tot }}=17.26 \pm 0.05$ for SAO $0822+3545$ using the growth curve method as described in Sect. 2.3. The respective integrated colours are $(B-V)_{\mathrm{tot}}=0.13$ and $(V-R)_{\mathrm{tot}}=0.17$. Relatively large errors originate from the zero-point uncertainties of the transformation equations (Kniazev et al. 2000). Accounting for a foreground extinction of $E(B-V)=0.047$ in our Galaxy (Schlegel et al. 1998), and applying the extinction curve from Whitford (1958), these colours become somewhat bluer: $(B-V)_{\mathrm{tot}}^{0}=0.08 \pm 0.06,(V-R)_{\mathrm{tot}}^{0}=0.14 \pm 0.06$.

The 2D spectrum in Fig. 2 and the irregular structure of the central part of SAO $0822+3545$ (see Fig. 3) indicate that enhanced star formation took place at least in the inner part of that galaxy. The possibility of better distinguishing individual regions of higher brightness (possible young superclusters and aged $\mathrm{H}$ II regions) in the central part of the LSBD was the motivation for using the MIDAS package IMRES for deconvolution of the NOT images. This package employs an image restoration scheme devised by Richardson and Lucy and described in Adorf et al. (1992), Hook \& Lucy (1992; and references therein). The number of iterations was determined by comparisons between the input image and the output deconvolved images, after convolution with the point-spread function (PSF).

The results of deconvolving the $B$-band image after 15 iterations are plotted in the right panel of Fig. 3. A number of filaments and relatively bright knots in the inner part of this LSB/dIrr galaxy are well resolved. Practically all of them are seen on the original image (left panel of Fig. 3), although deconvolution makes the structure more visible. All these structures are easily visible in a deconvolved $V$-band image, but are less distinct in $R$-band.

There is a faint tail in the SE direction in the outer part of the LSBD. This tail is visible on both blue DSS-II and $B$-band NOT images. The surface brightness of this tail corresponds to $\mu_{B}=25.7 \operatorname{arcsec}^{-2}$, or $2 \sigma$ of the noise level for the $B$-band image. The tail direction coincides with that of the LSBD major axis. The major axis PA varies from $-75^{\circ}$ for the inner part of the galaxy up to $-60^{\circ}$ for the outer part. The axial ratio is essentially constant over the body: $b / a \sim 0.55$.

Evidence for recent star-formation activity over the internal part of this dIrr galaxy is also seen, e.g., in the $(B-V)_{0}$ colour map (Fig. 4). This map shows a rather uniform distribution (mainly in the range from -0.05 to +0.10 ) over a large part of the main body. Its maximum $(+0.2$ to +0.25$)$ and minimum $(-0.15$ to -0.10$)$ values are measured in the positions close to the edges, where the $\mathrm{S} / \mathrm{N}$ is small, and these large colour variations are apparently spurious. 

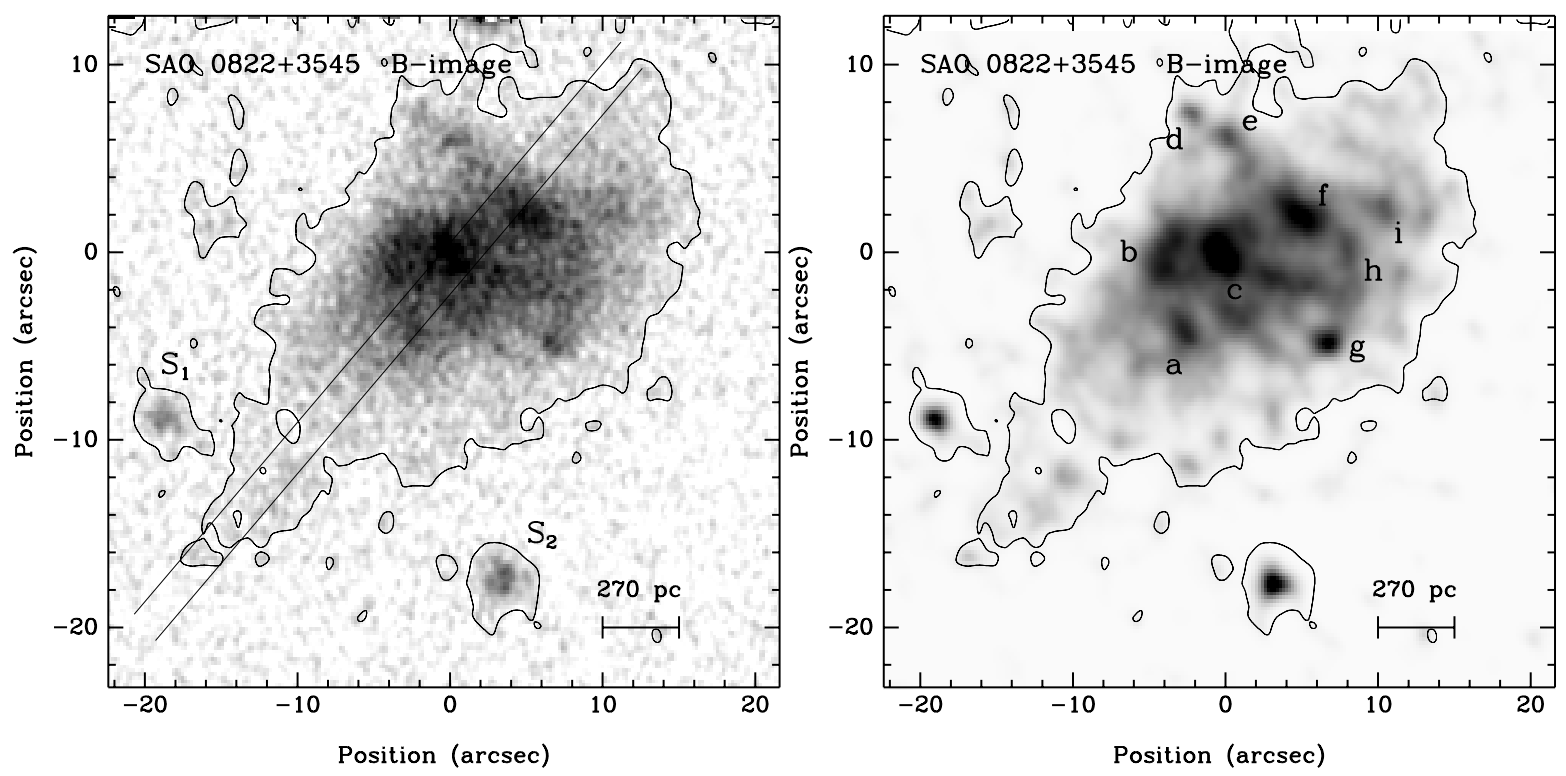

Fig. 3. Left panel: $B$-band image of SAO $0822+3545$ from the NOT. $\mathrm{N}$ is up, $\mathrm{E}$ is to the left. The $2^{\prime \prime}$-wide long slit of the $6 \mathrm{~m}$ telescope spectrum is superimposed. Highly irregular structure is seen in the inner $\sim 15^{\prime \prime}$. Two knots near the center and two arcs to the $\mathrm{N}$ and $\mathrm{W}$ are indicative of some activity in this galaxy. The isophotal contour corresponds to a $B$-band SB of $\sim 25.7 \mathrm{arcsec}^{-2}$, or $2 \sigma$ of background (for 2D Gaussian smoothing with $\left.F W H M=1^{\prime \prime}\right)$. Two faint objects, probable background galaxies in the vicinity of SAO $0822+3545$, are marked as $\mathrm{S}_{1}$ and $\mathrm{S}_{2}$. Right panel: The same $B$-band image of SAO 0822+3545, deconvolved after 15 iterations using the FFT based Lucy algorithm (Adorf et al. 1992). The angular resolution has improved from 1."25 to $\sim 0^{\prime} 85$ (FWHM). Several filaments and many knots are easily visible within the brighter part of the galaxy. Knots are marked by letters from "a" to "i", respectively.

Table 2. Photometric parameters of SAO $0822+3545$.

\begin{tabular}{cccc}
\hline \hline Band & $\begin{array}{c}\text { Total } \\
\text { mag } \\
(1)\end{array}$ & $\begin{array}{c}r_{\text {eff }} \\
\operatorname{arcsec}\end{array}$ & $\begin{array}{c}S B_{\text {eff }} \\
\mathrm{mag} \mathrm{arcsec}^{-2}\end{array}$ \\
\hline$B$ & $17.56 \pm 0.05$ & 6.46 & 23.60 \\
$V$ & $17.43 \pm 0.03$ & 6.43 & 23.46 \\
$R$ & $17.26 \pm 0.05$ & 7.94 & 23.75 \\
\hline
\end{tabular}

SAO $0822+3545$ is a genuine LSB galaxy with traces of recent $\mathrm{SF}$ near its center. Since the central bright knots are seen with the lowest contrast in $R$-band, we made a rough estimate of the central SB of an underlying "disk" in $R$. From the data in Table 2, its effective surface brightness corrected for extinction $\left(A_{R}=0.12\right)$ is $\mu_{\mathrm{eff}, 0}^{R}=23.63 \operatorname{arcsec}^{-2}$. For a purely exponential disk this corresponds to a central brightness of $\mu_{0}^{R}=22.50 \mathrm{arcsec}^{-2}$. Since the galaxy is significantly inclined to the line-of-sight, we need to make a corresponding correction to $\mu_{0}^{R}$. For an observed axial ratio $p=b / a=0.55$, assuming an intrinsic axial ratio of $q=0.2$, and using the well-known formula $\cos ^{2}(i)=\left(p^{2}-q^{2}\right) /\left(1-q^{2}\right)$, we calculate that $i=58.5^{\circ}$. The inclination correction for surface brightness is then $-2.5 \cdot \log (\cos i)=0.7$, giving a corrected central brightness of $\mu_{0, \mathrm{c}}^{R}=23 \mathrm{~m} .20 \operatorname{arcsec}^{-2}$. Even if the underlying "disk" is as blue, as the integrated light of this galaxy (that is, $(B-R)_{0} \sim 0.2$ ), its central brightness in $B$-band $\mu_{0, \mathrm{c}}^{B}=23.40 \operatorname{arcsec}^{-2}$ is well within the LSB galaxy regime. For an exponential law, $r_{\mathrm{eff}}=7.94^{\prime \prime}$ in $R$ corresponds to the scalelength $\alpha_{\mathrm{R}}=4.73^{\prime \prime}$.

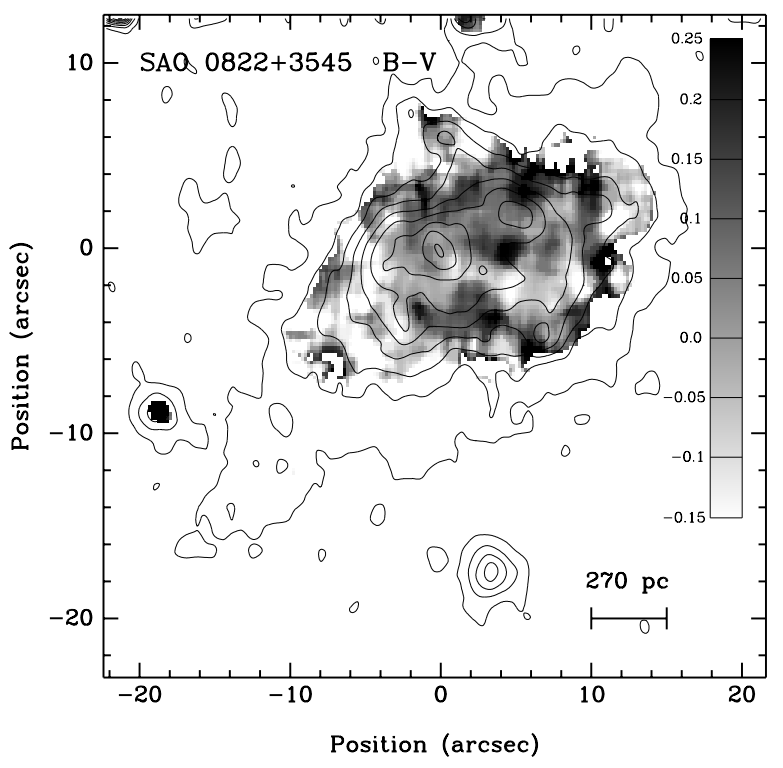

Fig. 4. Grey-scale map of $(B-V)$-colour for SAO $0822+3545$ smoothed with $2 \mathrm{D}$ median with the window size of $11^{\prime \prime} 6 \times 11^{\prime \prime} 6$. The extinction corrected $(E(B-V)=0.047)$ colour distribution is shown only for regions with $\mathrm{S} / \mathrm{N}$ ratio larger than 4 . The colour scale is shown in the right side column. $B$-band surface brightness (SB) isolines are superimposed, with the lowest SB level of $25.7 \mathrm{arcsec}^{-2}$. The other lev-

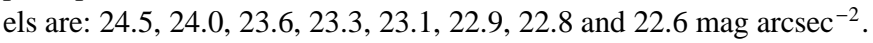

\subsection{Morphology and ionized gas motions of HS $0822+3542$}

We also applied the image restoration method to the NOT images of HS $0822+3542$. After 40 iterations the angular 

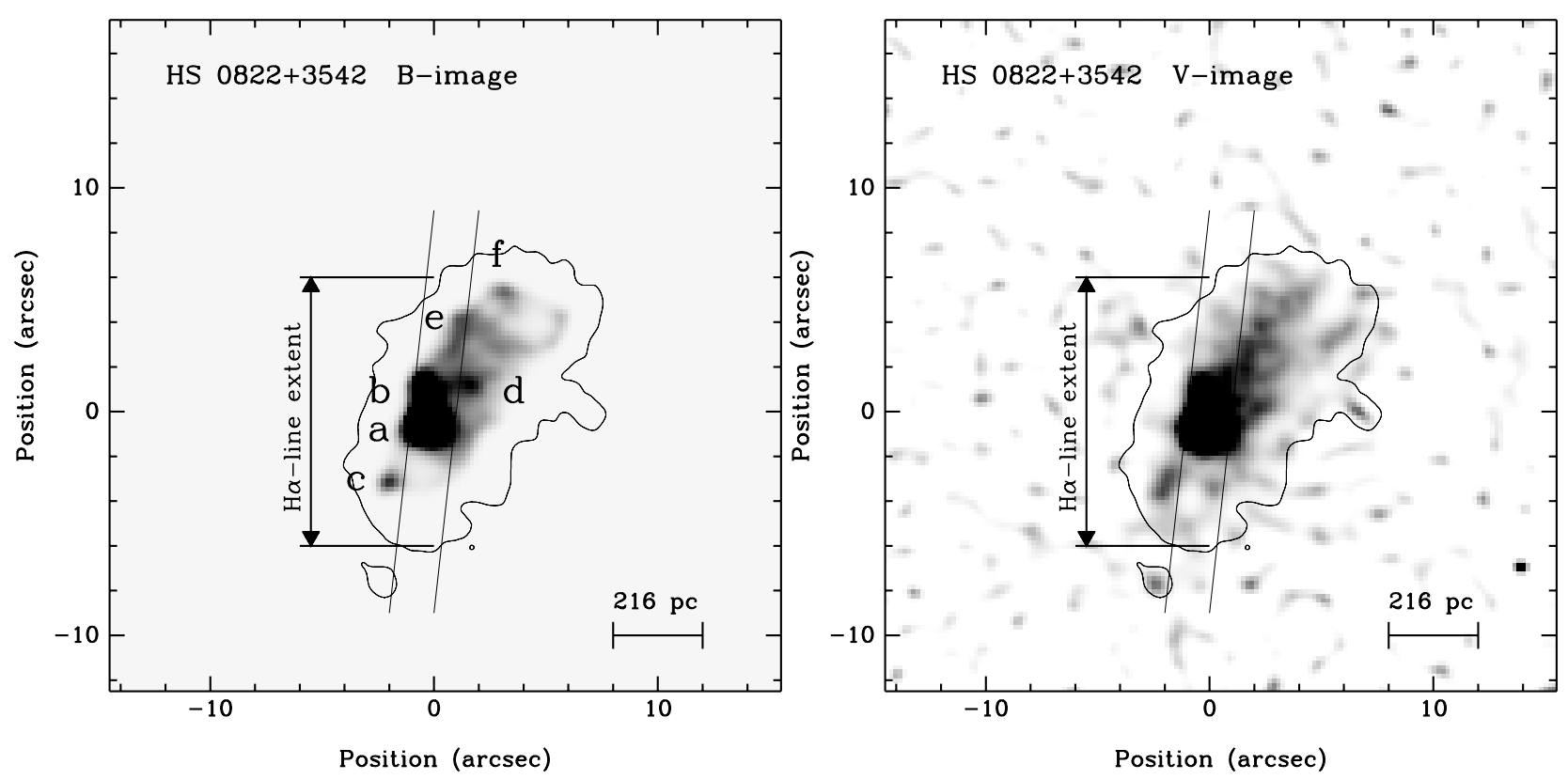

Fig. 5. Left panel: the NOT $B$-band image of HS $0822+3542$, deconvolved with 40 iterations using FFT based Lucy algorithm. The angular resolution is improved from 1". 25 to $\sim 0$ ' $^{\prime} 6 \mathrm{~N}$ is up, $\mathrm{E}$ is to the left. The slit position for $\mathrm{H} \alpha$ velocity curve is superimposed. The isophotal contour corresponds to $\mu_{\mathrm{B}}=25 \cdot 0 \operatorname{arcsec}^{-2}$ or $4 \sigma$ of background (for $2 \mathrm{D}$ Gaussian smoothing with $F W H M=1^{\prime \prime}$ ). The arrow indicates the extent of $\mathrm{H} \alpha$ emission on the $6 \mathrm{~m}$ telescope spectrum. All features marked as "a" to "f" are resolved in $B, V, R$ deconvolved images. Knot "b" is $\sim 8$ time fainter than "a" (see Sect. 3.3). Right panel: same as before, but for NOT $V$-band image. The filamentary structure on NW edge is well seen on both images.

resolution of the $B$-band image was improved by a factor of two $\left(F W H M=0{ }^{\prime} 6\right)$, and the restored BCG complex morphology is shown in Fig. 5 in grey scale, with a contour indicating the outer isophote $\mu_{\mathrm{B}}=25 \mathrm{~m} .0 \operatorname{arcsec}^{-2}$ superimposed. With the letters " $\mathrm{a}$ " to "f" we have marked all bright features well resolved after deconvolution in each of $B, V$, and $R$ bands. The brightest region consists of two components, "a" and " $b$ ", separated by $\sim 1.3^{\prime \prime}(\sim 70 \mathrm{pc})$, roughly in the $\mathrm{N}-\mathrm{S}$ direction. The contrast in Fig. 5 is adjusted to show faint features. The real ratio of the intensities of knots "a" and " $b$ ", derived from this image, is $\sim 8$. While deconvolution does not preserve brightness proportions between individual features, we consider this ratio to be indicative of the real value.

In addition to several bright knots, some filamentary structures are also visible. Some traces of them are also seen in other filters, but due to their lower surface brightness they are not as easily visible as the knots. Nevertheless, the prominent arc-like structure at the NW edge of the main body (stretching from $X=+2, Y=+4$ to $X=+6, Y=+4$ in Fig. 5), is clearly visible on both $B$ and $V$ images, but is more noisy in $R$-band, presumably due to the lower $\mathrm{S} / \mathrm{N}$ ratio. This feature probably represents an ionized gas shell with a diameter of $\sim 4^{\prime \prime}(\sim 200 \mathrm{pc})$, caused by recent active SF in this region, Four more knots are seen in the restored image. One ("d") is near the geometrical center of the main body. This, perhaps, might naturally be expected because of the gravitational well in the center of the galaxy. The others knots provide further evidence that the current SF episode in the BCG is spread across the galaxy.

The P-V diagram in Fig. 6 indicates a supershell with a size of $\sim 480 \mathrm{pc}$, comparable to the extent of $\mathrm{H} \alpha$-emission in the long-slit direction. The supershell velocity amplitude, as seen from our data, is about $30 \mathrm{~km} \mathrm{~s}^{-1}$. Such supershells are well-resolved on high-resolution $\mathrm{H} \alpha$ long-slit spectra for many nearby starbursting galaxies (e.g., Marlowe et al. 1995; Martin 1996, 1997, 1998). The related supershells of neutral gas are also seen in H I maps of such galaxies (e.g., Walter \& Brinks 1999). For the typical case of a starburst off-set in position relative to the midplane of the gas disk, the asymmetry of the gas density distribution in the z-direction results in the shell's asymmetric appearance. This was shown, e.g., in numerical simulations by Silich et al. (1996), and by Walter \& Brinks (1999) for the observed types of P-V diagrams. That part of the shell propagating out of the plane should have a significantly lower Emission Measure due to decreasing gas density, and usually appears much fainter in comparison to the part of the shell moving towards the midplane (e.g., Martin 1996).

Shells (or supershells) are produced by hot bubbles, caused by the injection of the energy from numerous massive star winds and supernova (SN) explosions into the interstellar medium (ISM) (e.g., Tenorio-Tagle \& Bodenheimer 1988). The asymmetric appearance of supershells in $\mathrm{H} \alpha$ emission is easily seen with the high-resolution data mentioned above. When the velocity resolution is not sufficient to distinguish motions on both sides of the shell, we measure the intensity-weighted velocity in the $\mathrm{H} \alpha$-line at each slit position. If the $\mathrm{H} \alpha$ intensities in the segments on opposite sides of the shell differ significantly, we will see mainly the side approaching the midplane of the gas disk. However, since the observed velocity at each slit position is the weighted mean of the emission from the opposite sides of the shell, a shell velocity derived in this way represents a lower limit of the real value. Depending on the relative strengths of 


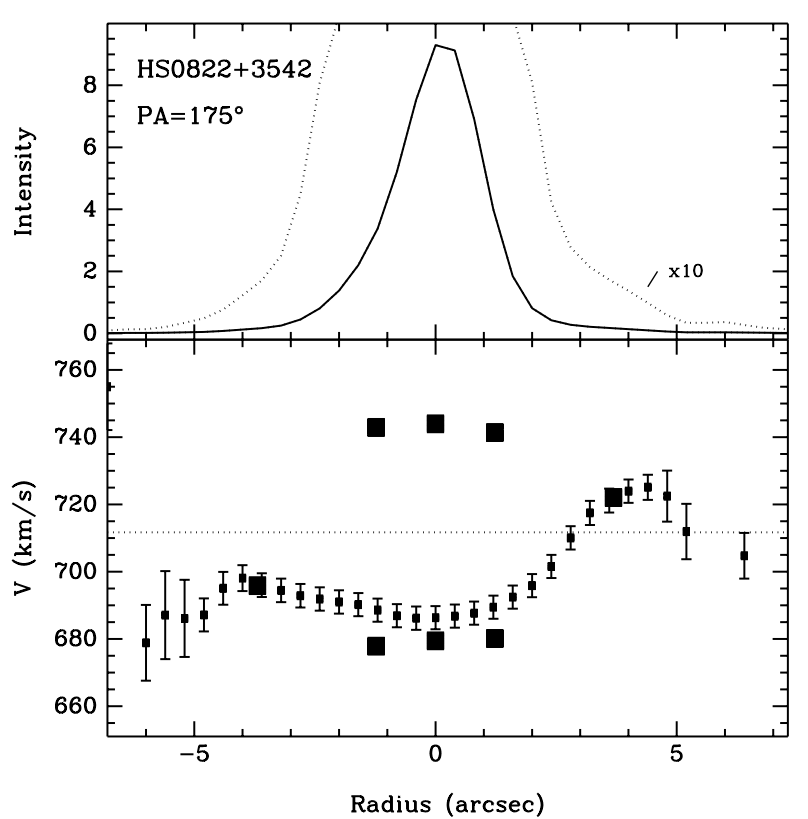

Fig. 6. Upper panel: $\mathrm{H} \alpha$ intensity in $\mathrm{HS} 0822+3542$ along the slit position shown in Fig. 5. Lower panel: P-V diagram of ionized gas in HS $0822+3542$ based on the $\mathrm{H} \alpha$ line from the same spectrum. Its form within a radius of $\pm 4^{\prime \prime}$, and the characteristic diameter and velocity amplitude $\left(\sim 480 \mathrm{pc}\right.$ and $\sim 30 \mathrm{~km} \mathrm{~s}^{-1}$ ), are consistent with the appearance of supershells observed in many star-bursting galaxies. Filled boxes show the velocities on both sides of the shell as derived from the Gaussian decomposition of the $\mathrm{H} \alpha$ line at several positions along the slit. For details see Sect. 3.3.

$\mathrm{H} \alpha$ emission on the shell sides the expected correction could reach tens of percent.

If we accept the full amplitude of the radial velocity difference between the two edges on the $\mathrm{P}-\mathrm{V}$ diagram $\left(\sim 35 \mathrm{~km} \mathrm{~s}^{-1}\right)$ as the result of rotation in the BCG, then the apparent $V_{\text {rot }}$ is $17.5 \mathrm{~km} \mathrm{~s}^{-1}$. With an apparent axial ratio $p=b / a=0.5$, and an assumed intrinsic ratio $q=0.2$, using the same relation as for SAO $0822+3545$ in Sect. 3.2 we get an inclination angle $i=63.6^{\circ}$, and thus an inclination-corrected value of $V_{\text {rot }}=19.5 \mathrm{~km} \mathrm{~s}^{-1}$. The latter value is quite consistent with that expected from the Tully-Fisher relation between galaxy $V_{\text {rot }}$ and its blue luminosity, namely, using the relations derived by Karachentsev et al. (1999) for dwarf galaxies in the Local Volume. For HS $0822+3542, M_{\mathrm{B}}=-12.5$ (or $L_{\mathrm{B}}=$ $\left.1.56 \times 10^{7} L_{\odot}\right)$ and so the expected $V_{\text {rot }}=17 \mathrm{~km} \mathrm{~s}^{-1}$, with a $\pm 1 \sigma$ confidence range of 12 to $24 \mathrm{~km} \mathrm{~s}^{-1}$.

In Fig. 6 we use filled squares to show the velocities on both sides of the shell, obtained by Gaussian decomposition of the $\mathrm{H} \alpha$ profiles. The two-component structure is detectable only for sufficiently large $\mathrm{S} / \mathrm{N}$ ratios and maximal velocity separation. The flux from the receding components is several times lower than that from the approaching ones. These data confirm the existence of a large shell with a characteristic velocity amplitude of $\sim 30 \mathrm{~km} \mathrm{~s}^{-1}$ and exclude the interpretation of this feature in the $\mathrm{P}-\mathrm{V}$ diagram as part of a rotation curve. Indeed, the rotation velocity should monotonously increase from one edge to another. The real $\mathrm{P}-\mathrm{V}$ diagram certainly does not look like this. Thus, if the ionized gas in the BCG is rotating, its rotation velocity is sufficiently small to be practically hidden by the visible large shell.

\subsection{Chemical abundances in HS $0822+3542$}

The spectrum of the brightest knot of HS $0822+3542$, extracted with an aperture of 3 .' $^{\prime} \times 11^{\prime \prime} 2$, is shown in Fig. 7. It is dominated by very strong emission lines. The relative intensities of all emission lines, together with the equivalent width $E W(\mathrm{H} \beta)$, extinction coefficient $C(\mathrm{H} \beta)$ and the $E W$ of Balmer absorption lines are given in Table 3. $C(\mathrm{H} \beta)$ was derived from the Balmer decrement using the self-consistent method of Izotov et al. (1994). The derived value of $C(\mathrm{H} \beta)=0.0 \pm 0.09$ is consistent, to within the uncertainties, with a reddening of $E(B-V)=0.047 \pm 0.007$, expected from foreground extinction in the Galaxy (Schlegel et al. 1998).

We analyzed chemical abundances and physical parameters with the method described by Kniazev et al. (2000). The measured electron temperatures and density, and derived chemical abundances are presented in Table 4. Our new data give a slightly higher value of $12+\log (\mathrm{O} / \mathrm{H})(7.44 \pm 0.06$ versus $7.35 \pm 0.04$ from the NOT spectrum), but the difference is not significant. The main source of this difference is the intensity of the [O III] $\lambda 4363$ line, relative to $\mathrm{H} \beta$. Its measured value is 0.104 on the $6 \mathrm{~m}$ telescope spectrum, in comparison to 0.123 on the NOT spectrum. Part of the derived differences in $\mathrm{O} / \mathrm{H}$ could be due to the differences in observational conditions, resulting in the sampling of slightly different regions. However, both results are in fact consistent to within their uncertainties. The ratios of other heavy element abundances $(\mathrm{Ne}, \mathrm{S}, \mathrm{N})$ to that of oxygen, derived from the NOT and the $6 \mathrm{~m}$ telescope data, are also similarly consistent. With the higher resolution (1.2 $\AA$ pixel $^{-1}$ ) spectrum near $\mathrm{H} \alpha$ we improved the precision of the $\mathrm{N}$ abundance, since the [N II] $\lambda 6584$ emission line was detected with a higher $\mathrm{S} / \mathrm{N}$ ratio than in the NOT spectrum. The intensities of Ar lines are measured for the first time in this BCG, and we present here its abundance. At the position of the [Fe III] line $\lambda 4658 \AA$ we detected a signal at a level of $4 \sigma$. However, if $\log (\mathrm{Fe} / \mathrm{O})$ is typical of other very metal-poor BCGs $(\sim-1.65)$, the signal in this line should be only $\sim 1.3 \sigma$. One possible explanation of the strength of this line is the contribution of the WR spectral feature C IV $\lambda$ 44658. In many BCGs with detected WR features the intensity of the C IV $\lambda 4658$ line is comparable to that of He II $\lambda 4686$ (e.g., Guseva et al. 2000). Thus, a likely interpretation of the observed feature is that it is the sum of the lines of [Fe III] and C IV. This suggests that higher $\mathrm{S} / \mathrm{N}$ spectroscopy could detect the other WR features in this young starburst region. The He II $\lambda 4686$ line in our spectrum is broadened to $\sim 15 \AA$, but the $\mathrm{S} / \mathrm{N}$ ratio is too low to accept this as direct evidence of a WR population.

\section{Discussion}

\subsection{General parameters of the system}

In Table 5 we present the main parameters relevant for further discussion of the properties and status of these dwarf galaxies. Their small mutual projected distance $(3.5$, or $\sim 11 \mathrm{kpc})$ 


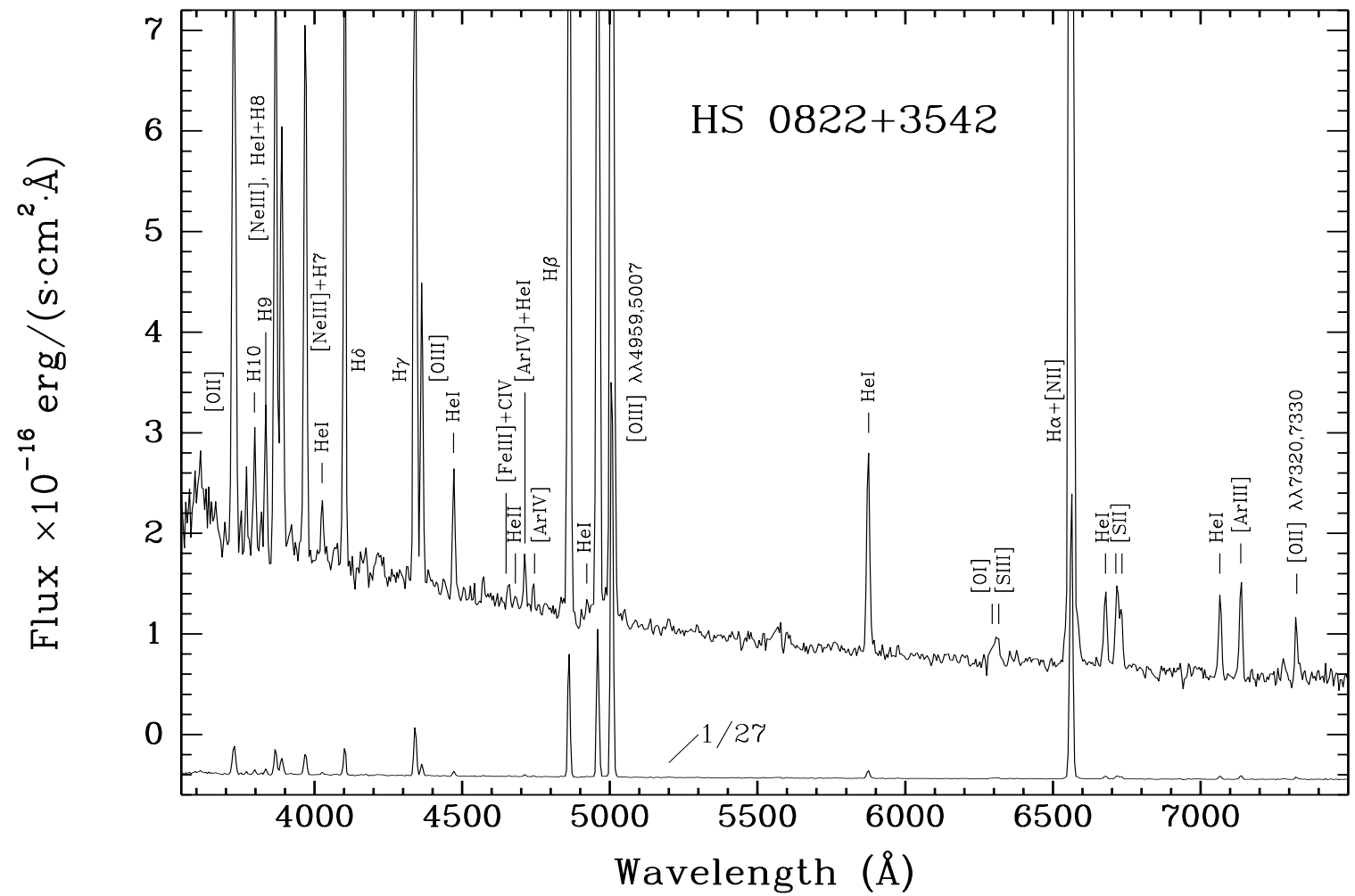

Fig. 7. 1D spectrum of HS $0822+3542$ with a sampling of $4.6 \AA$ pixel $^{-1}$. The spectrum in the bottom is scaled by $1 / 27$ to show the relative intensities of strong lines.

and relative velocity $\left(\Delta V<25 \mathrm{~km} \mathrm{~s}^{-1}\right.$, Chengalur et al. 2003) imply that both galaxies are physically associated. Some of the parameters for HS $0822+3542$ in Table 5 have been revised from those in Kniazev et al. (2000). In particular, the distance-dependent parameters have changed due to an improved distance estimate. H I related parameters have also changed due to correction of the 21-cm line flux (GMRT, Chengalur et al. 2003). The integrated H I flux presented by Kniazev et al. (2000), based on observations with the NRT, appeared to be off by a factor of two due to the effects of confusion with the galaxy SAO $0822+3545$. The adopted oxygen abundance is the weighted mean of current and previous (Kniazev et al. 2000) values.

\subsection{On the evolutionary status of $S A O 0822+3545$}

\subsubsection{The very blue colours of SAO $0822+3545$}

The integrated colours of SAO $0822+3545$ are unusually blue. Only two out of about 250 LSB/dIrr galaxies with known integrated colours $(B-V),(V-R)$, or $(B-R)$ (from papers by Ronnback \& Bergvall 1994; McGaugh \& Bothun 1994; de Blok et al. 1995; van Zee et al. 1997; O'Neil et al. 1997; van Zee et al. 2001; Burkholder et al. 2001) have such blue colours. Only one of the 65 dIrr galaxies studied by Makarova et al. (1998), Makarova \& Karachentsev (1998) and Makarova (1999) appeared that blue; Makarova et al. (1998) noted that this particular LSBG (UGCA 292) has several blue stellar complexes, in which van Zee (2000) detected strong line emission, indicating young starbursts.
Thus, the unusual colours of SAO $0822+3545$ could be due to its recent enhanced SF. To estimate the age of its stellar population, we can compare its $(B-V)_{\text {tot }}^{0}$ and $(V-R)_{\text {tot }}^{0}$ colours with model values. Unfortunately, due to the age-metallicity degeneracy, similar colours can correspond to very different ages. Therefore some a priori information on galaxy metallicity is necessary to disentangle the degeneracy. This can be obtained from the dIrr galaxy metallicity-luminosity relation (Skillman et al. 1989; Pilyugin 2001). For SAO 0822+3545 it predicts $Z \sim 1 / 20 Z_{\odot}$. In fact, for LSB galaxies this relation likely goes significantly below (e.g., Kunth \& Östlin 2000) that from Skillman et al., so $1 / 20 Z_{\odot}$ is probably the upper limit for the metallicity of SAO $0822+3545$.

\subsubsection{Comparison of observed and model parameters}

To get some insight on the evolution status of SAO $0822+3545$, we compared its observed colours and $E W(\mathrm{H} \alpha)$ with model predictions. For colours we used results derived from PEGASE.2 models (Fioc \& Rocca-Volmerange 1997, 2000). We also used these models to estimate the mass of the stellar population. We calculated spectral energy distributions (SEDs) for instantaneous SF bursts with $Z=1 / 20$ and $1 / 50 Z_{\odot}$, as well as the time behavior of $B, V, R$ luminosities and the respective colour tracks. In Fig. 8, we show $B V R$ colour tracks for instantaneous SF bursts with $Z=1 / 20$ and $1 / 50 Z_{\odot}$, using solid and dotted lines, respectively, assuming a Salpeter IMF with $M_{\text {low }}=0.1 M_{\odot}, M_{\text {up }}=120 M_{\odot}$. The track for continuous SF with constant SFR $\left(Z=1 / 20 Z_{\odot}\right)$ and the same IMF is shown by dashed line. SAO $0822+3545$ extinction corrected 
Table 3. Line intensities of HS $0822+3542$.

\begin{tabular}{|c|c|c|}
\hline$\lambda_{0}(\AA)$ Ion & $F(\lambda) / F(\mathrm{H} \beta)$ & $I^{a}(\lambda) / I(\mathrm{H} \beta)$ \\
\hline $3727[\mathrm{O}$ II $]$ & $0.3116 \pm 0.0239$ & $0.3061 \pm 0.0255$ \\
\hline 3868 [Ne III] & $0.2381 \pm 0.0224$ & $0.2339 \pm 0.0232$ \\
\hline $3889 \mathrm{He}$ I $+\mathrm{H} 8$ & $0.1599 \pm 0.0178$ & $0.1846 \pm 0.0219$ \\
\hline $3967[\mathrm{Ne} \mathrm{III]}]+\mathrm{H} 7$ & $0.1966 \pm 0.0145$ & $0.2217 \pm 0.0180$ \\
\hline $4026 \mathrm{He} \mathrm{I}$ & $0.0209 \pm 0.0036$ & $0.0205 \pm 0.0036$ \\
\hline $4101 \mathrm{H} \delta$ & $0.2281 \pm 0.0170$ & $0.2495 \pm 0.0199$ \\
\hline $4340 \mathrm{H} \gamma$ & $0.4413 \pm 0.0316$ & $0.2495 \pm 0.0199$ \\
\hline $4340 \mathrm{H} \gamma$ & $0.4413 \pm 0.0316$ & $0.4567 \pm 0.0340$ \\
\hline 4363 [O III] & $0.1044 \pm 0.0079$ & $0.1026 \pm 0.0080$ \\
\hline $4471 \mathrm{He} \mathrm{I}$ & $0.0390 \pm 0.0036$ & $0.0383 \pm 0.0036$ \\
\hline 4658 [Fe III $]+$ C IV & $0.0081 \pm 0.0021$ & $0.0080 \pm 0.0021$ \\
\hline $4686 \mathrm{He}$ II & $0.0053 \pm 0.0015$ & $0.0080 \pm 0.0021$ \\
\hline $4686 \mathrm{He}$ II & $0.0053 \pm 0.0015$ & $0.0052 \pm 0.0015$ \\
\hline 4713 [Ar IV] + He I & $0.0158 \pm 0.0031$ & $0.0155 \pm 0.0031$ \\
\hline 4740 [Ar IV] & $0.0071 \pm 0.0021$ & $0.0070 \pm 0.0021$ \\
\hline $4861 \mathrm{H} \beta$ & $1.0000 \pm 0.0720$ & $1.0000 \pm 0.0734$ \\
\hline 4959 [O III] & $1.2104 \pm 0.0877$ & $1.1889 \pm 0.0877$ \\
\hline 5007 [O III] & $3.6425 \pm 0.2637$ & $3.5779 \pm 0.2639$ \\
\hline $5876 \mathrm{He}$ I & $0.0852 \pm 0.0064$ & $0.0837 \pm 0.0066$ \\
\hline $6300[\mathrm{OI}]$ & $0.0082 \pm 0.0029$ & $0.0081 \pm 0.0029$ \\
\hline 6312 [S III] & $0.0099 \pm 0.0028$ & $2.7510 \pm 0.2172$ \\
\hline $6584[\mathrm{~N} \mathrm{II}]$ & $0.0105 \pm 0.0029$ & $0.0098 \pm 0.0028$ \\
\hline $6563 \mathrm{H} \alpha$ & $2.7842 \pm 0.1990$ & $2.7510 \pm 0.2172$ \\
\hline 6584 [N II] & $0.0105 \pm 0.0029$ & $0.0103 \pm 0.0029$ \\
\hline $6678 \mathrm{He} \mathrm{I}$ & $0.0282 \pm 0.0038$ & $0.0277 \pm 0.0039$ \\
\hline 6717 [S II] & $0.0268 \pm 0.0037$ & $0.0263 \pm 0.0038$ \\
\hline $6731[\mathrm{~S} \mathrm{II}]$ & $0.0182 \pm 0.0034$ & $0.0179 \pm 0.0034$ \\
\hline $7065 \mathrm{He} \mathrm{I}$ & $0.0168 \pm 0.0042$ & $0.0165 \pm 0.0043$ \\
\hline 7136 [Ar III] & $0.0244 \pm 0.0035$ & $0.0239 \pm 0.0036$ \\
\hline$C(\mathrm{H} \beta) \operatorname{dex}$ & \multicolumn{2}{|c|}{$0.00 \pm 0.09$} \\
\hline$E W($ abs $) \AA$ & \multicolumn{2}{|c|}{$4.90 \pm 0.67$} \\
\hline$E W(\mathrm{H} \beta) \AA$ & \multicolumn{2}{|c|}{$271 \pm 14$} \\
\hline
\end{tabular}

colours (with their $\pm 1 \sigma$ uncertainties) are also plotted in the figure as the filled triangle.

The $B V R$ colours of SAO $0822+3545\left((B-V)_{0}=0.08 \pm\right.$ $\left.0.06,(V-R)_{0}=0.14 \pm 0.06\right)$ fall very close to the model tracks. For instantaneous SF with $Z=1 / 20 Z_{\odot}$ they correspond to an age of $\sim 110\left( \pm_{80}^{50}\right)$ Myr. For continuous SF with a constant rate the respective age is $\sim 0.6\left( \pm_{0.3}^{0.7}\right)$ Gyr. Thus, the integrated colours of this galaxy allow various interpretations of its evolutionary status.

To further constrain the range of the models, consistent with the very blue $B V R$ colours of SAO $0822+3545$ we use two additional parameters: the observed $E W(\mathrm{H} \alpha)$ and the dynamic mass of this galaxy. To model the observed $E W(\mathrm{H} \alpha)$ we did not use PEGASE.2, since a coherent computation of stellar and nebular emission would require coupling with the CLOUDY code (Ferland 1996; Moy et al. 2001). Instead, we compared values of $E W(\mathrm{H} \alpha)$ with the predictions of Starburst99 (SB99, Leitherer et al. 1999). In Table 6 we summarize all the models we have examined in order to match both the observed $B V R$ and $E W(\mathrm{H} \alpha)$. Each considered model also predicts a total stellar mass, which should be compatible with $M_{\mathrm{dyn}}$. Since LSBGs
Table 4. Abundances in HS $0822+3542$.

\begin{tabular}{lc}
\hline \hline$T_{\mathrm{e}}(\mathrm{O}$ III $)(\mathrm{K})$ & $18200 \pm 1000$ \\
$T_{\mathrm{e}}(\mathrm{O} \mathrm{II})(\mathrm{K})$ & $15100 \pm 800$ \\
$T_{\mathrm{e}}(\mathrm{S} \mathrm{III})(\mathrm{K})$ & $16800 \pm 800$ \\
$N_{\mathrm{e}}(\mathrm{S} \mathrm{II})\left(\mathrm{cm}^{-3}\right)$ & $\leq 10$ \\
& \\
$\mathrm{O}^{+} / \mathrm{H}^{+}\left(\times 10^{5}\right)$ & $0.259 \pm 0.040$ \\
$\mathrm{O}^{++} / \mathrm{H}^{+}\left(\times 10^{5}\right)$ & $2.478 \pm 0.345$ \\
$\mathrm{O} / \mathrm{H}\left(\times 10^{5}\right)$ & $2.737 \pm 0.348$ \\
$12+\log (\mathrm{O} / \mathrm{H})$ & $7.44 \pm 0.06$ \\
& \\
$\mathrm{~N}^{+} / \mathrm{H}^{+}\left(\times 10^{7}\right)$ & $0.753 \pm 0.178$ \\
$\mathrm{ICF}(\mathrm{N})$ & 10.566 \\
$\log (\mathrm{N} / \mathrm{O})$ & $-1.54 \pm 0.12$ \\
& \\
$\mathrm{Ne}$ & \\
$\mathrm{ICF}(\mathrm{Ne})$ & 1.105 \\
$\log (\mathrm{Ne} / \mathrm{O})$ & $-0.87 \pm 0.09$ \\
& \\
$\mathrm{~S}^{+} / \mathrm{H}^{+}\left(\times 10^{+}\right)$ & $0.430 \pm 0.059$ \\
$\mathrm{~S}^{++} / \mathrm{H}^{+}\left(\times 10^{7}\right)$ & $3.569 \pm 1.091$ \\
$\mathrm{ICF}(\mathrm{S})$ & 2.542 \\
$\log (\mathrm{S} / \mathrm{O})$ & $-1.43 \pm 0.13$ \\
& \\
$\mathrm{Ar} \mathrm{H}^{++} / \mathrm{H}^{+}\left(\times 10^{7}\right)$ & $0.713 \pm 0.114$ \\
$\mathrm{Ar}{ }^{+++} / \mathrm{H}^{+}\left(\times 10^{7}\right)$ & $0.660 \pm 0.210$ \\
$\mathrm{ICF}(\mathrm{Ar})$ & 1.008 \\
$\log (\mathrm{Ar} / \mathrm{O})$ & $-2.30 \pm 0.09$ \\
\hline
\end{tabular}

are known as gas-rich galaxies, it is worthwhile to compare this stellar mass with the total gas mass $M_{\text {gas }}$ (see the estimates of $M_{\text {dyn }}$ and $M_{\text {gas }}$ in Sect. 4.2.3).

We will only briefly discuss the models from Table 6 which fail to match the three observational parameters, and then turn to a couple of more realistic models. First, simple scenarios with constant SFR and ages in the range of 0.4-1.1 Gyr, for the standard Salpeter IMF (Models 4 and 5, as the representatives of the whole range of ages), should be excluded, since the predicted $E W(\mathrm{H} \alpha)$ is much higher then the observed value of 15 to $20 \AA$. Similarly, Model 3, with the same IMF, an instantaneous SF episode, and an age of $\sim 100 \mathrm{Myr}$, is excluded due to the very small expected $E W(\mathrm{H} \alpha)$.

Models 6 and 7 deal with IMFs biased to the low-mass end. For a continuous SF law, lasting $\sim 250$ and $\sim 100 \mathrm{Myr}$, respectively (shown in Fig. 8 by empty stars), they have $B V R$ colours consistent either with those observed, or with colours corrected for a possible extinction $E(B-V) \sim 0.10$. $E W(\mathrm{H} \alpha)$, according to SB99, appears to be consistent with the observed values. However, the mass of formed stars $M_{\text {star }}$, as estimated with PEGASE.2, is several times larger than the LSBG dynamic mass (see Sect. 4.2.3). Therefore both Model 6 and 7 should be rejected.

More realistic models include composite stellar populations created by instantaneous SF episodes with the standard Salpeter IMF. Indeed, since a single-component Model 3 fails to reproduce the observed $E W(\mathrm{H} \alpha)$, we would need a younger Single Stellar (coeval) Population (SSP), to give enough ionizing photons. But this would appear too blue, and in order 
Table 5. Main parameters of the studied galaxies.

\begin{tabular}{|c|c|c|}
\hline Parameter & HS $0822+3542$ & SAO 0822+3545 \\
\hline$\overline{\mathrm{RA}(\mathrm{J} 2000.0)}$ & 082555.47 & 082605.59 \\
\hline $\operatorname{Dec}(\mathrm{J} 2000.0)$ & +353232.9 & +353525.7 \\
\hline$A_{\mathrm{B}}($ from NED) & 0.20 & 0.20 \\
\hline$B_{\text {tot }}$ & $17.92 \pm 0.05^{(1)}$ & $17.56 \pm 0.03^{(2)}$ \\
\hline$(B-V)_{\mathrm{tot}}^{0}$ & $0.27 \pm 0.07^{(1)}$ & $0.08 \pm 0.06^{(2)}$ \\
\hline$(V-R)_{\mathrm{tot}}^{0}$ & $0.14 \pm 0.09^{(1)}$ & $0.14 \pm 0.06^{(2)}$ \\
\hline$V_{\text {hel }}(\mathrm{H} \mathrm{I})\left(\mathrm{km} \mathrm{s}^{-1}\right)$ & $716^{(8)}$ & $738^{(8)}$ \\
\hline $\operatorname{Dist}^{(3)}(\mathrm{Mpc})$ & 11.0 & 11.0 \\
\hline$M_{\mathrm{B}}^{0}(4)$ & -12.49 & -12.85 \\
\hline Opt. size $\left({ }^{\prime \prime}\right)^{5}$ & $14.8 \times 7.4^{(1)}$ & $28.2 \times 15.5^{(2)}$ \\
\hline Opt. size (kpc) & $0.79 \times 0.39$ & $1.50 \times 0.83^{(2)}$ \\
\hline $12+\log (\mathrm{O} / \mathrm{H})$ & $7.38^{(1,2)}$ & - \\
\hline H I int. flux ${ }^{(6)}$ & $0.34^{(8)}$ & $0.98^{(8)}$ \\
\hline$M(\mathrm{H} \mathrm{I})\left(10^{7} M_{\odot}\right)$ & $0.97^{(2)}$ & $2.8^{(2)}$ \\
\hline$M(\mathrm{HI}) / L_{\mathrm{B}}^{(7)}$ & $0.63^{(2)}$ & $1.31^{(2)}$ \\
\hline
\end{tabular}

(1) - From Kniazev et al. (2000).

(2) - Derived in this paper.

(3) - Distance derived according to the kinematics of the Local Volume (Karachentsev \& Makarov 2001).

(4) - Corrected for Galactic extinction $A_{B}$.

(5) $-a \times b$ at $\mu_{\mathrm{B}}=25^{\mathrm{m}} \cdot 0 \operatorname{arcsec}^{-2}$.

(6) - In units of Jy.km s${ }^{-1} ;(7)-$ in units of $\left(M / L_{\mathrm{B}}\right)_{\odot}$.

(8) - From Chengalur et al. (2003).

to reproduce the integrated $B V R$ colours of SAO $0822+3545$, one would need to prepare a mixture with many older redder stars. For illustration, we show in Fig. 8 the colours of a series of composite models with filled squares. They consist of various mixtures of two coeval populations (SSP) with ages of $t_{1}=10 \mathrm{Myr}$ and $t_{2}=10 \mathrm{Gyr}$. Their mass ratio $\eta$ varies as explained in the figure caption. Model 1 in Table 6 with $\eta=30$ best matches both $B V R$ colours and $E W(\mathrm{H} \alpha)$, and its $M_{\text {star }}$ is compatible with $M_{\text {dyn }}$ and $M_{\text {gas }}$. The third SSP component (in parentheses) with an age of $\sim 100 \mathrm{Myr}$ and a mass comparable to that of the 10-Myr old component could also be present, however it is not necessary for a good fit to the observed data.

Another series of composite models is shown in Fig. 8 by open circles. These models consist of two coeval populations with ages of $t_{1}=10 \mathrm{Myr}$ and $t_{2}=250 \mathrm{Myr}$. The model with $\eta=10$, which best matches the observed $B V R$ and $E W(\mathrm{H} \alpha)$, is Model 2 in Table 6. Again, $M_{\text {star }}$ for this model is consistent with $M_{\text {dyn }}$ and $M_{\text {gas }}$.

Such types of composite models with two coeval populations, one of which has an age $t_{1}=10 \mathrm{Myr}$, can be constructed for any $t_{2}$ in the range between $\sim 250 \mathrm{Myr}$ and $\sim 10 \mathrm{Gyr}$, and $\eta$ between $\sim 10$ and $\sim 30$. All of them provide good matches to the observed colours and $E W(\mathrm{H} \alpha)$, and have stellar masses compatible with the dynamic and gas mass of the LSBD.

Finally, we discuss one more model, with an IMF deficit in massive stars relative to the "standard" IMF used in the discussion above. This IMF has $\alpha=2.35$, and $M_{\text {up }}$ and $M_{\text {low }}$ of 30 and $0.1 M_{\odot}$, respectively. According to SB99 (Fig. 84), a model with this IMF and continuous SF (Model 8) will reach the observed value of $E W(\mathrm{H} \alpha)=20 \AA$ in $\sim 1$ Gyr. The PEGASE. 2

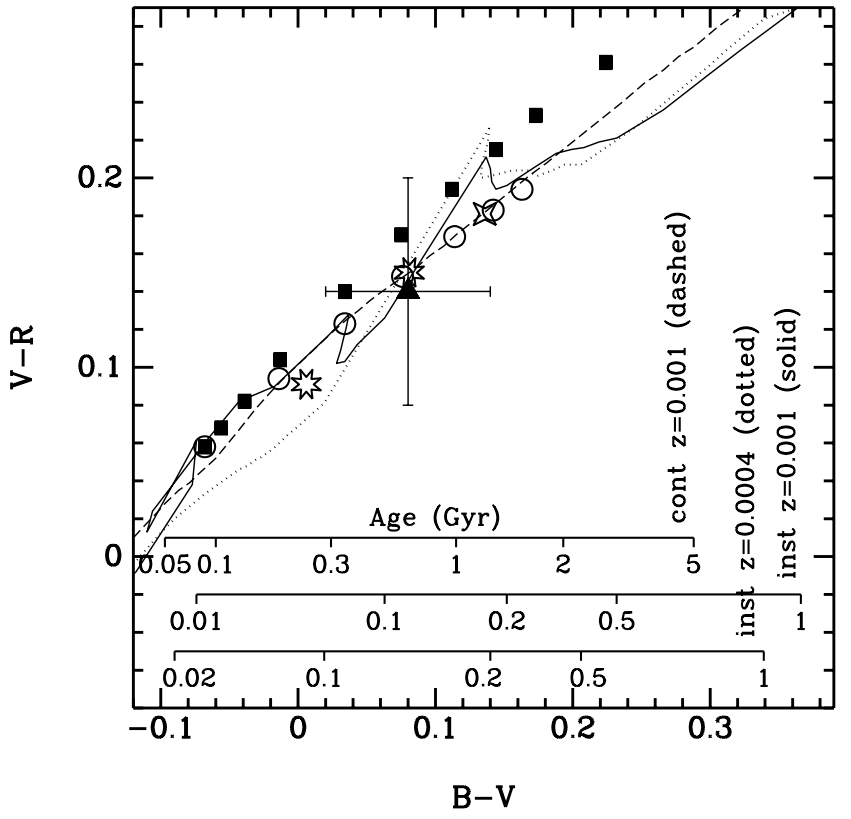

Fig. 8. $B V R$ tracks based on PEGASE.2 evolution models from Fioc $\&$ Rocca-Volmerange (1997). Solid and dotted lines are for instantaneous starburst stellar populations with $Z$ of $1 / 20 Z_{\odot}$ and $1 / 50 Z_{\odot}$, respectively (Salpeter IMF, $M_{\text {up }}$ and $M_{\text {low }}$ are 120 and $0.1 M_{\odot}$ ). The dashed line is for $1 / 20 Z_{\odot}$ continuous $\mathrm{SF}$ at a constant rate. Time scales (in Gyr) for respective tracks are shown in the bottom. The filled triangle with error bars shows corrected for Galactic extinction of $E(B-V)=0.047$ the total $B V R$ colours of SAO $0822+3545$. Filled squares show the sequence of $B V R$ colors for composite stellar populations with a "young" (10 Myr) starburst and an admixture of a 10 Gyr ("old") coeval population. The mass ratio $\eta=M_{\text {old }} / M_{\text {young }}$ varies along the sequence as $0,2,5,10,20,30,40,50,60$ and 80 . Open circles show the colours of a composite population with the same $10 \mathrm{Myr}$ "young" population mixed with a $250 \mathrm{Myr}$ ("old") coeval population. For this case the mass ratio $\eta$ sequence is shown for values $0,2,5,10,20,40$ and 80 . Two open stars show the $B V R$ colours of a stellar population resulting from continuous SF with a steep IMF ( $\alpha=3.35$ ), lasting 100 and $250 \mathrm{Myr}$, while the cross shows the colours for the case of continuous SF, lasting $1 \mathrm{Gyr}$, but for a Salpeter IMF with $M_{\text {up }}=30 M_{\odot}$ (see text).

colours of such a model are $(B-V)=0.14$ and $(V-R)=0.20$ (open cross in Fig. 8). They are compatible with the observed colours to within their uncertainties. The resulting $M_{\text {star }}$ is also compatible with the LSBG $M_{\text {dyn }}$ and $M_{\text {gas }}$.

Summarizing this point, we conclude that for a Salpeter IMF with $M_{\text {up }}=120 M_{\odot}$ only a model with a composite population can explain both the photometry and the $E W(\mathrm{H} \alpha)$ data. It has to include a "young" coeval population with an age of $\sim 10 \mathrm{Myr}$ and an "old" coeval component. If the age of the "old" population is $10 \mathrm{Gyr}$, it should be $\sim 30$ times more massive than the "young" component; an "old" component with $T \sim 250$ Myr should be 10 times more massive than the "young" one. The real situation is likely somewhere in between these two extreme cases. In any case, for an "old" component with an age between 0.25 and $10 \mathrm{Gyr}$, its mass should be between 10 and 30 times the masses of the "young" population. An "intermediate" age ( 100 Myr) coeval population, 
Table 6. Parameters of various evolution models vs. those observed in SAO 0822+3545.

\begin{tabular}{|c|c|c|c|c|c|c|}
\hline $\begin{array}{l}\text { Model IMF } \\
\text { (1) }\end{array}$ & Model Nu. & $\begin{array}{l}\text { SF type } \\
\text { \& Age } \\
\text { (3) }\end{array}$ & $\begin{array}{c}\text { Mass } \\
\text { (in } M_{\odot} \text { ) } \\
\text { (4) }\end{array}$ & $\begin{array}{c}(B-V)^{0} \\
(5)\end{array}$ & $\begin{array}{c}(V-R)^{0} \\
(6)\end{array}$ & $\begin{array}{c}E W(\mathrm{H} \alpha) \\
\text { (in } \AA \text { ) } \\
(7)\end{array}$ \\
\hline \multirow[t]{5}{*}{$\begin{array}{l}\alpha=2.35 \\
0.1-120 M_{\odot}\end{array}$} & 1 & $\begin{array}{l}\mathrm{SSP}_{1} t_{1}=10 \mathrm{Myr} \\
\mathrm{SSP}_{2} t_{2}=10 \mathrm{Gyr} \\
\left(\mathrm{SSP}_{3} t_{3}=100 \mathrm{Myr}\right)\end{array}$ & $\begin{array}{c}4.4 \times 10^{5} \\
1.3 \times 10^{7} \\
\left(4.4 \times 10^{5}\right)\end{array}$ & 0.07 & 0.17 & 20 \\
\hline & 2 & $\begin{array}{l}\mathrm{SSP}_{1} t_{1}=10 \mathrm{Myr} \\
\mathrm{SSP}_{2} t_{2}=250 \mathrm{Myr}\end{array}$ & $\begin{array}{l}2.4 \times 10^{5} \\
2.4 \times 10^{6}\end{array}$ & 0.07 & 0.15 & 20 \\
\hline & 3 & $\mathrm{SSP}_{1} t_{1}=100 \mathrm{Myr}$ & $2.5 \times 10^{6}$ & 0.06 & 0.13 & $<0.1$ \\
\hline & 4 & $\operatorname{Con}_{1} t_{1}=0.4 \mathrm{Gyr}$ & $1.2 \times 10^{8}$ & 0.04 & 0.12 & 100 \\
\hline & 5 & $\mathrm{Con}_{2} t_{2}=1.1 \mathrm{Gyr}$ & $7.8 \times 10^{7}$ & 0.13 & 0.20 & 150 \\
\hline \multirow{2}{*}{$\begin{array}{l}\alpha=3.35 \\
0.1-120 M_{\odot}\end{array}$} & 6 & $\mathrm{Con}_{1} t_{1}=250 \mathrm{Myr}$ & $2.0 \times 10^{9}$ & 0.08 & 0.15 & 20 \\
\hline & 7 & $\mathrm{Con}_{2} t_{2}=100 \mathrm{Myr}$ & $4.3 \times 10^{9}$ & 0.01 & 0.09 & $20^{a}$ \\
\hline $\begin{array}{l}\alpha=2.35 \\
0.1-30 M_{\odot}\end{array}$ & 8 & $\operatorname{Con}_{1} t_{1}=1 \mathrm{Gyr}$ & $8.7 \times 10^{7}$ & 0.14 & 0.18 & 20 \\
\hline $\begin{array}{l}\text { Observed in } \\
\text { SAO } 0822+3545\end{array}$ & & $\begin{array}{l}M_{\mathrm{gas}}= \\
M_{\mathrm{dyn}}=\end{array}$ & $\begin{array}{l}3.7 \times 10^{7} \\
4-7 \times 10^{8} \\
\end{array}$ & $\begin{array}{r}0.08 \\
\pm 0.06 \\
\end{array}$ & $\begin{array}{c}0.14 \\
\pm 0.06 \\
\end{array}$ & 20 \\
\hline
\end{tabular}

${ }^{a} B V R$ colours correspond to those observed, after correction for possible internal extinction of $E(B-V)=0.1$.

having model $B V R$ colours close to those observed for $\mathrm{SAO}$ $0822+3545$, could also contribute appreciable mass.

Some uncertainties of these estimates are related to the respective uncertainties of the LSBD metallicity and/or uncorrected internal extinction. The variation of the former parameter by a factor of two will slightly change the relative mass of "young" and "old" stellar components. The internal extinction is usually small in LSB galaxies. For example, the galaxy corrected $B V R$ colours of the LSBD would still lie close to the model evolution tracks even with a correction for an internal extinction of $E(B-V)=0.10$. However, these colours would correspond to a significantly smaller age, $\sim 30$ Myr. The selfconsistent interpretation of the galaxy colours and observed $E W(\mathrm{H} \alpha)$ still requires a composite model, similar to those discussed above. The main effect of such reddening (if present) would be the significant reduction (by a factor of 4-5) of the mass ratio, $\eta$.

For the IMF with a deficit of massive stars, both $E W(\mathrm{H} \alpha)$ and $B V R$ colours can be explained by a single component with a constant SFR and an age of $\sim 1$ Gyr. The formed stellar mass would be consistent with $M_{\text {dyn }}$. Note, however, that such a model would be inconsistent with the recent encounter of the LSBD and BCG, and its resulting disturbance (Sect. 4.5). In addition, internal extinction on the level of $E(B-V)=0.10$ would cause problems for such a model, since this case would require ages of only $100-250 \mathrm{Myr}$, an $E W(\mathrm{H} \alpha)$ that is too large $(\gtrsim 50 \AA)$, and $M_{\text {star }} \sim M_{\text {dyn }}$.

The available data on the integrated $B V R$-colours and $E W(\mathrm{H} \alpha)$ in SAO $0822+3545$ imply that the significant or main stellar mass fraction is related to young populations with ages from 10 Myr to 1 Gyr. In case of a "standard" Salpeter IMF with $M_{\text {up }}=120 M_{\odot}$, this LSBG likely experienced at least two localized SF episodes. Continuous SF, lasting $\sim 1 \mathrm{Gyr}$, can match all available data with a single population, if the IMF is significantly biased to the low-mass end. To distinguish which of the two SF scenarios is more probable, we would need to measure the integrated $B V R$ colours of SAO $0822+3545$ with better precision $\left(\sigma \lesssim 0^{\mathrm{m}} 02\right)$, and determine the amount of internal extinction.

\subsubsection{The LSBD gas mass fraction}

The ratio of gas and stellar masses is one of the important parameters characterizing the evolutionary status of the galaxy. The gas-mass fraction (relative to the full visible mass) of several XMD BCGs reaches values of 0.94-0.98. This is a good indication of their possible youth. In addition, Schombert et al. (2001), in a recent study of a large sample of LSBGs, discovered several galaxies with gas-mass fractions of $0.8-0.85$. Perhaps these are the galaxies with the lowest known SFRs 
Table 7. Nearby voids parameters.

\begin{tabular}{lrrrrrrrrr}
\hline \hline Designation & $\begin{array}{r}\text { Size } \\
\mathrm{km} \mathrm{s}^{-1}\end{array}$ & $\begin{array}{r}\text { RA } \\
\text { hour }\end{array}$ & $\begin{array}{r}\text { Dec } \\
\circ\end{array}$ & $\begin{array}{r}c z \\
\mathrm{~km} \mathrm{~s}^{-1}\end{array}$ & $\begin{array}{r}l^{\mathrm{II}} \\
{ }^{\circ}\end{array}$ & $\begin{array}{r}b^{\mathrm{II}} \\
\circ\end{array}$ & $\begin{array}{r}\text { SGX } \\
\mathrm{km} \mathrm{s}^{-1}\end{array}$ & $\begin{array}{r}\text { SGY } \\
\mathrm{km} \mathrm{s}^{-1}\end{array}$ & $\begin{array}{r}\text { SGZ } \\
\mathrm{km} \mathrm{s}^{-1}\end{array}$ \\
\hline Cetus & 500 & 2.0 & -20 & 700 & 192 & -72 & 100 & -600 & -200 \\
Cepheus & 500 & 23.5 & +65 & 800 & 112 & 5 & 700 & 0 & 300 \\
Crater & 500 & 11.5 & -15 & 1500 & 126 & -28 & 1300 & -700 & 200 \\
Volans & 700 & 7.0 & -70 & 800 & 281 & -25 & -600 & -300 & -500 \\
Lynx-Cancer & 800 & 8.0 & +30 & 800 & 191 & 27 & 400 & 400 & -500 \\
Monoceros & 1000 & 4.0 & +05 & 800 & 185 & -34 & 400 & -400 & -500 \\
\hline
\end{tabular}

and/or relatively young objects. To estimate the masses of various populations in SAO $0822+3542$ we used its $B$-band luminosity. We also assumed $Z=1 / 20 Z_{\odot}$ and considered the PEGASE. 2 models, presented in Table 6.

The full gas mass of the LSBD is derived directly from $\mathrm{H} \mathrm{I}$ data in Table 5, accounting for a helium mass-fraction of 0.24 : $M_{\text {gas }} \sim 3.7 \times 10^{7} M_{\odot}$. Then, for "Model 1" (10 Gyr "old" stellar population $)$, the gas mass fraction is $f_{\mathrm{g}} \equiv M_{\mathrm{gas}} /\left(M_{\mathrm{gas}}+M_{\mathrm{star}}\right)$ $=0.73$. For "Model 2' (the "old" stellar population $0.25-\mathrm{Gyr}$ old), the gas mass fraction is $f_{\mathrm{g}}=0.93$. For the LSBD "old" coeval population with an age between 0.25 and $10 \mathrm{Gyr}$, the gas mass fraction will thus fall in the range of 0.73 to 0.93 . For "Model 8" with continuous SF, the gas mass fraction would be only $\sim 0.3$. While the most probable SF scenario for this LSBD remains somewhat uncertain, it could be one of the most gasrich galaxies of its type.

One more parameter, important for comparison with models, is the total dynamic mass of galaxy. From the NRT HI profile of Kniazev et al. (2000), in which the LSBD contributes more than half of the flux, we get an upper limit of $V_{\text {rot }}$ of $20 \mathrm{~km} \mathrm{~s}^{-1}$. Corrected for an inclination angle of $58.5^{\circ}$ (Sect. 3.2) this yields $V_{\text {rot }}$ of $23.5 \mathrm{~km} \mathrm{~s}^{-1}$. For the commonly assumed gas disk radius of 3-4 times $R_{\mathrm{opt}}$, this results in $M_{\text {dyn }}=(4-7) \times 10^{8} M_{\odot}$.

\subsection{Global environment: The Lynx-Cancer Void}

HS $0822+3542$ is well-isolated with regard to known, sufficiently bright galaxies. There are no cataloged galaxies in the NED and LEDA databases with $V_{\text {hel }}<1200 \mathrm{~km} \mathrm{~s}^{-1}$ and angular distances less than $5.8^{\circ}$. All the nearest cataloged galaxies (at projected distances of 1.15-1.45 Mpc) are dwarfs with $M_{\mathrm{B}}$ in the range -13.5 to -16.6 . The position of HS $0822+3542$ and SAO $0822+3545$ is shown (in the box) in Fig. 9. The positions of other cataloged galaxies with radial velocities of $V_{\text {hel }}=V_{\text {pair }} \pm 300 \mathrm{~km} \mathrm{~s}^{-1}( \pm 4 \mathrm{Mpc})$ are shown by filled circles, where $V_{\text {pair }}=727 \mathrm{~km} \mathrm{~s}^{-1}$ is the mean velocity of the pair. Absolute magnitudes of all galaxies in the plot (corrected for Galactic extinction according to Schlegel et al. 1998) are derived from their cataloged magnitudes. The corrections for Local Group motion, and the value of the local Hubble constant $\left(67.5 \mathrm{~km} \mathrm{~s}^{-1} \mathrm{Mpc}^{-1}\right)$ in this direction were made according to Karachentsev \& Makarov (2001).
There are six bright galaxies $\left(M<M_{\mathrm{B}}^{*}=-19.73\right)$ in the plot area. The latter corresponds to a volume element with sides (at $D=11 \mathrm{Mpc}$ ) of $9.5 \mathrm{Mpc} \times 7.8 \mathrm{Mpc}(\mathrm{RA} \times \mathrm{Dec})$, and $\sim 9 \mathrm{Mpc}$ in depth. All of them (shown by the largest filled circles) are situated at the three-dimensional distances from the dwarf pair greater than 3.0 Mpc. Seven intermediate luminosity galaxies with $-19.73 \leq M_{\mathrm{B}}<-17.73$ (shown by slightly smaller circles), are also situated along the periphery of this volume. Only a few real dwarfs are found inside this very large region. With regard to bright galaxies, the pair of dwarfs in question is situated in a void, not far from the void center. The void depth in radial velocity through its center is of order $\sim 800 \mathrm{~km} \mathrm{~s}^{-1}$ (from 400 to $1200 \mathrm{~km} \mathrm{~s}^{-1}$, or $\sim 12 \mathrm{Mpc}$ ). This is comparable to its projected extent on the sky. This void is similar to the other nearby voids discussed by Fairall (1998). We have named it the Lynx-Cancer void, for the constellations on which this void is projected. The parameters of this and the other similar voids are presented in Table 7. The latter are taken from Fairall's (1998) Table 4.1. The first column gives the void's designation. In Cols. 2, 3 and 4 the radial velocity extent, and the approximate equatorial coordinates of the void's center are given. In Col. 5 we present the void's central heliocentric velocity. Columns 6 and 7 give the galactic longitude and latitude of the void's center. Finally, Cols. 8, 9 and 10 present the supergalactic coordinates.

Understanding the properties of galaxy populations in voids is important for both galaxy and structure formation models. It is natural to expect that candidate truly young galaxies would be very isolated (e.g., Peebles 2001). Observationally, however, the situation is unclear due to very poor statistics. For example, SBS 0335-052, one of the best candidate young galaxies, is situated at the outskirts of a loose galaxy group. It is interesting to note, however, that the recent data indicate that at least several new extremely metal-deficient (XMD) BCGs $\left(Z \lesssim 1 / 20 Z_{\odot}\right)$, including HS $0822+3542$, and HS 2236+1344 (Ugryumov et al. 2003), indeed lie in regions of very low (massive) galaxy density. A larger sample of XMD BCGs would be helpful for determining whether they really favour a void-type environment.

\subsection{Supershell and young clusters in HS $0822+3542$}

From the parameters of the supershell, we can estimate its characteristic age, and thus, the time elapsed since the SF episode 


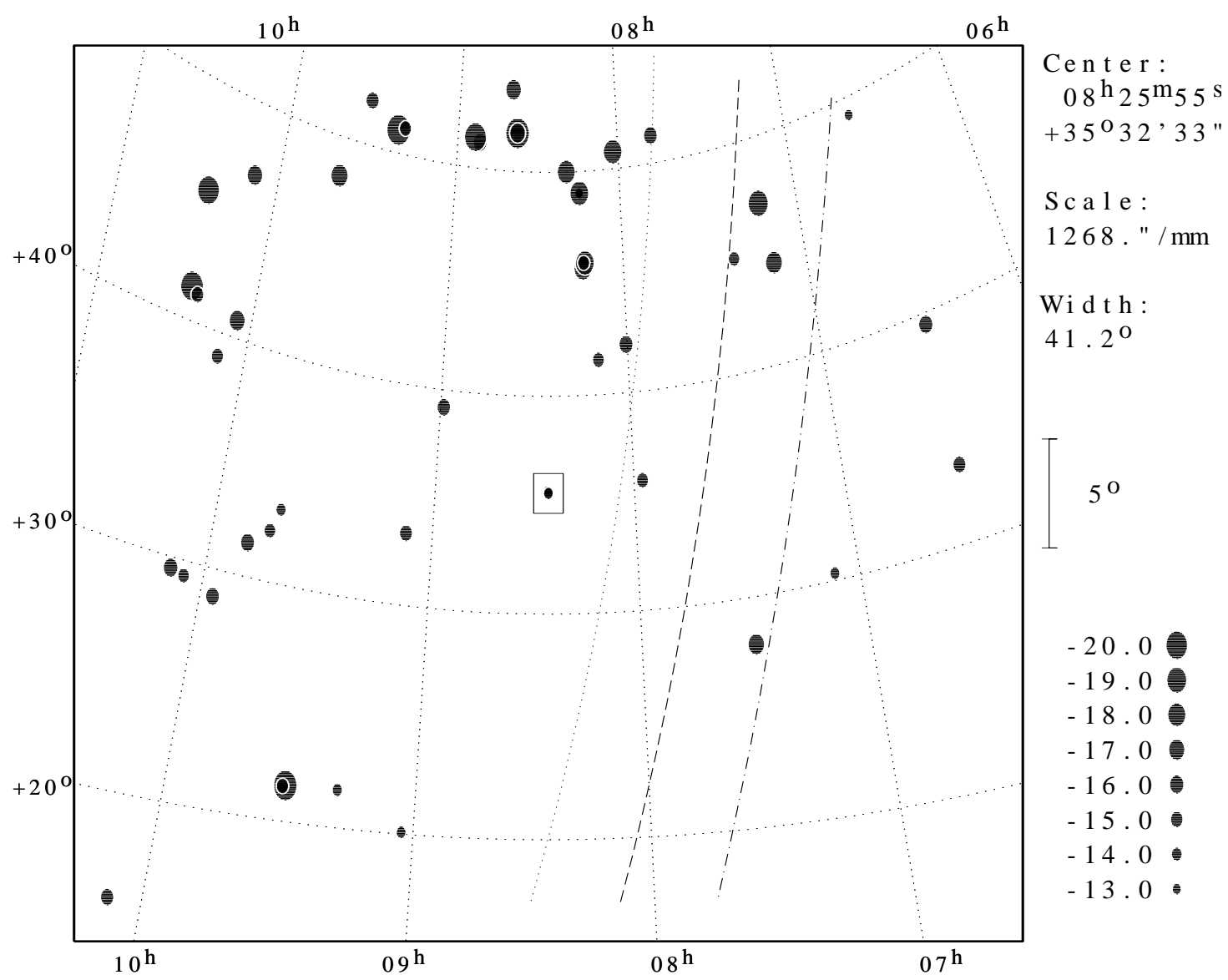

Fig. 9. The position of HS $0822+3542$ and SAO $0822+3545$ (in the box) is shown relative to the other galaxies from LEDA in the redshift range between $V_{\text {hel }}=427$ and $1027 \mathrm{~km} \mathrm{~s}^{-1}$. The blue luminosities of galaxies are indicated by the size of the filled circle. The nearest neighbour to the pair, at the position RA $\sim 8^{\mathrm{h}}$, DEC $\sim 36^{\circ}(D=1.15 \mathrm{Mpc})$ has $M_{\mathrm{B}}=-14.7$. Dotted, dashed and dot-dashed lines show the projection of galactic latitudes $b^{\mathrm{II}}=30^{\circ}, 25^{\circ}$ and $20^{\circ}$, respectively. Galactic extinction should more strongly affect the detection of faint galaxies. The absence of bright galaxies is not a selection effect, since some of them are found in the zone of $b^{\mathrm{II}}<25^{\circ}$,

which produced this supershell. The relation between the shell age, its velocity and radius, for the case in which the radiative cooling of hot gas inside the shell can be neglected (readily applicable for multiple SN-blown bubbles), is given as $t_{\text {shell }}=0.6 \times R / V_{\text {shell }}$ (e.g., Weaver et al. 1977; Mac Low \& McCray 1988). This relation also holds approximately for the thin shell phase, in which a bubble is pumped by a stationary stellar wind. The observed values are $V_{\mathrm{obs}}=30 \mathrm{~km} \mathrm{~s}^{-1}$ and $R=0.24 \mathrm{kpc}$. Both the maximal velocity and the apparent size of the shell in a simple case depend similarly on the inclination angle. Thus, no inclination correction is necessary. The shell age is then $t_{\text {shell }} \sim 5 \mathrm{Myr}$.

The age of the current SF burst can be estimated from the observed $E W(\mathrm{H} \beta)=271 \AA$ (see Table 3 ) using the results of instantaneous SF burst models (Leitherer et al. 1999). For a Salpeter IMF with $M_{\text {low }}$ and $M_{\text {up }}$ of 1 and $100 M_{\odot}$, respectively, this value of $E W(\mathrm{H} \beta)$ corresponds to a starburst age of $\sim 3$ Myr. It is important to note that both the main part of the starburst continuum and the highly concentrated $\mathrm{H} \beta$ emission of the corresponding $\mathrm{H}$ II region were extracted for the analyzed spectrum.

Hence, the expected corrections for $E W(\mathrm{H} \beta)$ are insignificant, and the estimate of starburst age is reliable. It is also worth noting that, for the Salpeter IMF models with $M_{\text {low }}=0.1 M_{\odot}$, the maximum value of $E W(\mathrm{H} \beta)$ is $\sim 230 \AA$, and, hence, these models cannot reproduce such a high $E W(\mathrm{H} \beta)$; this could be due to a "top-heavy" IMF in this object.

For further analysis, we assume, that SN explosions turn on in 3.5 Myr after the starburst beginning (e.g., Leitherer 1994; Leitherer \& Heckman 1995; Fioc \& Rocca-Volmerange 1997). The energy injected by stellar winds should also be considered when calculating shell parameters. For example, for $Z=Z_{\odot}$ the kinetic energies injected by winds and by SNe during the first $5 \mathrm{Myr}$ are comparable. However, the former scales as $Z^{+1}$, and for HS $0822+3542\left(Z \sim 1 / 30 Z_{\odot}\right)$ the energy injected by winds will be much lower than that of SNe. Accounting for SNe delay and the shell age, we conclude that the active SF in this region of HS $0822+3542$ has lasted at least 8-9 Myr. Hence, the current SF episode is extended, probably with propagating SF.

The shell kinetic energy, $E_{\text {kin }}$, can be estimated simply from its radius and velocity, suggesting some reasonable value of swept gas density. For $V_{\text {shell }}=30 \mathrm{~km} \mathrm{~s}^{-1}, R=0.24 \mathrm{kpc}$ and an average density of the ambient gas of $0.1 \mathrm{~cm}^{-3}, E_{\mathrm{kin}}$ will be as following: $E_{\text {kin }}=0.5 \mathrm{M} \cdot V^{2}=1.3 \times 10^{51} \mathrm{erg}$. Accounting for the small efficiency of SN energy transfer to the kinetic energy of the shell, $\sim 0.1$ (e.g., Thornton et al. 1998), 
the total energy of multiple SNe pumping the observed BCG supershell is $\sim 1.3 \times 10^{52} \mathrm{erg}$. This corresponds to the energy of 13 typical SNe. Taking the dynamical age of the supershell to be $5 \mathrm{Myr}$, we get a SNe rate of $2.6 \mathrm{SNe} \mathrm{Myr}^{-1}$. For a Salpeter IMF with $M_{\text {low }}$ and $M_{\text {up }}$, respectively, of 0.1 and $120 M_{\odot}$, from comparison with PEGASE. 2 models, translates this to a total coeval star cluster mass of $\sim 1.0 \times 10^{4} M_{\odot}$. The $M_{B}$ of such a cluster with an age of $8.5 \mathrm{Myr}$ would be -8.8 . At the distance of HS $0822+3542$, the apparent $B$ magnitude of this cluster should be $B=21.6$. The latter is $\sim 2 \mathrm{~m} 2$ (or a factor of 7) fainter than the estimate of $B=19.46$ found for the current starburst component of HS $0822+3542$ by Kniazev et al. (2000, Table 4, Col. 8). However, this value is quite consistent with the intensity of knot "b", seen in the deconvolved image of HS $0822+3542$ just $\sim 1.3^{\prime \prime}$ to the north of the main starburst region "a". Recall that its intensity is estimated as $\sim 1 / 8$ of that for knot "a" in Sect. 3.3. Thus, knot "b" could be the star cluster responsible for the observed ionized gas supershell. The younger star cluster (knot "a") is potentially the source of gas ionization in the shell. Its mass is estimated from $B_{\text {burst }}=19.46$, corresponding to $M_{B}^{0}=-10.95$. From the PEGASE. 2 model with the same IMF as above, a 3-Myr old starburst with $M_{B}^{0}=-10.95$ would have the mass of $3.9 \times 10^{4} M_{\odot}$.

It is possible that the shell age is slightly overestimated, while the age of the young starburst ( 3 Myr) could be underestimated, so that they could be more or less consistent with each other. In this case it is worth considering the alternative option: that the shell is pumped by this starburst. Then only the energy of stellar winds is available. According to Weaver et al. (1977), the kinetic energy of the shell comprises only about $20 \%$ of the total wind energy released during its lifetime. This implies a required wind energy of $6.5 \times 10^{51} \mathrm{erg}$. The temporal behavior of stellar wind kinetic luminosity is given in SB99. As an example, this behavior is illustrated in Fig. 13 of Efremov et al. (2002) for the case of a $10^{6} M_{\odot}$ young star cluster with $M_{\text {low }}=1 M_{\odot}$ and $Z=Z_{\odot}$. The estimate of the full wind kinetic energy released by such cluster during the first $3 \mathrm{Myr}$ is $9.9 \times 10^{53} \mathrm{erg}$. For $M_{\text {low }}=0.1 M_{\odot}$, this energy is scaled down by a factor of 2.5 . The wind power scales with metallicity as $Z^{+1}$. Thus, for the case of HS $0822+3542$, that wind energy, scaled down by a factor of 30 , yields a value of $3.3 \times 10^{52} \mathrm{erg}$ (or $1.3 \times 10^{52} \mathrm{erg}$ for $M_{\text {low }}=0.1 M_{\odot}$ ). That is, to provide the above required wind kinetic energy one would need a 3-Myr old star cluster with a full mass (depending on $M_{\text {low }}$ ) of (25) $\times 10^{5} M_{\odot}$ and $Z=1 / 30 Z_{\odot}$. Then, with the PEGASE. 2 model for $Z=1 / 20 Z_{\odot}$ (the nearest to that of HS $0822+3542$ ) we find that such a star cluster would have the blue luminosity, corresponding to $M_{B}=0.52$ per $1 M_{\odot}$. Respectively, for masses of $(2-5) \times 10^{5} M_{\odot}$ the cluster expected absolute magnitudes are in the range of $M_{B}=-12.7$ to -13.7 . These are significantly brighter than the observed $M_{B}=-10.95$ of the current young starburst. Therefore, this option does not look plausible.

We also revise the apparent stellar mass of the underlying "disk". Based on the published value $B_{\text {disk }}=18.22$ (Kniazev et al. 2000), its $M_{B}^{0}$ is -12.19 . According to the same PEGASE. 2 model, for a starburst with an age of $100 \mathrm{Myr}$ we have $M_{B}=3.13$ per $1 M_{\odot}$. The "disk" stellar mass is then $\sim 1.3 \times 10^{6} M_{\odot}$. Finally, we derive the BCG gas mass-fraction, $f_{\mathrm{g}}=0.90$, using for $M(\mathrm{HI})$ from Table 5 and a helium mass fraction of 0.24 . The significant difference from the value in Kniazev et al. (2000) is a result of the reduced $M(\mathrm{HI})$, as explained in Sect. 4.1.

\subsection{Triggering mechanisms of SF activity of the pair}

The SF history of LSB galaxies is a complicated issue. The suggested options range from SF episodes induced by distant encounters (O'Neil et al. 1998) to low-level sporadic SF, migrating across the galaxy body. The latter case can be considered as a continuous process with the time-scale on the order of a galaxy's life-time (Gerritsen \& de Blok 1999). Since the pair of dwarf galaxies discussed here is situated deeply in a void, any interactions with massive galaxies on a cosmological timescale would be very unlikely.

On the other hand, the importance of interactions with low-mass neighbours to trigger starbursts in BCG/H II-galaxies has been noted, e.g., by Taylor et al. (1995), Pustilnik et al. (2001b), and Noeske et al. (2001). The example of the BCG HS $0822+3542$ indicates that, even in voids, SF bursts can be triggered by the same mechanism (see also Pustilnik et al. 2002).

The projected distance between the dwarfs, $11.4 \mathrm{kpc}$, and their relative radial velocity, $\Delta V \leq 25 \mathrm{~km} \mathrm{~s}^{-1}$ (Chengalur et al. 2003), are both very small. This implies rather slow collision, which is very efficient in exerting mutual tidal forces. The N-body simulation of a non-merging encounter between LSB and HSB (high surface brightness) disk galaxies presented by Mihos et al. (1997) shows qualitatively different effects in each of the two galaxy types. Due to the lower surface density of baryonic matter and the stabilizing role of the Dark Matter (DM) halo throughout the galaxy, LSBGs are more stable to many internal and external perturbations. Thus, only a weak spiral wave is typically generated in a LSBG. This leads to some localized non-central low-level SF, and, hence, to no appreciable changes of galaxy properties. In contrast, in a HSB disk galaxy the strong perturbation of gas orbits leads to global instability, resulting in the disturbed gas sinking into the center of gravitational well (where it should eventually collapse and cause a burst of SF).

The situation for this particular pair of gas-rich dwarfs is quite reminiscent of these simulations. Since the LSBD is significantly more massive than the BCG, the mutual effect of their encounter will be enhanced for the BCG, and weaker for the LSBD. However, currently available data on the properties of these galaxies are too limited to make a more detailed comparison with galaxy interaction models. $\mathrm{HI}$ and $\mathrm{H} \alpha$ mapping will help to determine some of their parameters (e.g., the full dynamic mass, gas density and SFR distribution), and thus allow realistic modelling of this pair.

An additional argument for the effect of mutual interactions on the enhanced SF in both galaxies is the fact of significant SF events in each galaxy occurred on approximately the same timescale, which is comparable to the characteristic times of the two galaxies. All of them are on the order of a few hundred Myr. For the LSBD, we have already discussed the ages of 
"old" component in composite models of its $B V R$ colours. For HS $0822+3542$ the age of the major SF episode is implied by the detected Balmer absorptions in the underlying stellar disk (Izotov et al., in preparation). The time elapsed since the pericenter passage and strongest interaction between the galaxies is approximately $\sim 200-300$ Myr. The development of instabilities in a disturbed galaxy is delayed by a time comparable to the revolution period. The latter is on the order of $100 \mathrm{Myr}$. So, to a first approximation, the time since the strongest interaction, the delay time for gas collapse, and the ages of the stellar populations in the underlying disks of the LSBD and the BCG all are comparable.

As an interacting galaxy, HS $0822+3542$ is similar to several other XMD BCGs. Clear evidence of tidal disturbance was found for the optical counterpart of the large HI cloud Dw 1225+0152 (Salzer et al. 1991; Chengalur et al. 1995). The huge H I cloud, hosting the pair of XMD BCGs SBS 0335-052 E and W, also looks quite disturbed (Pustilnik et al. 2001a). The synchronized SF episodes in the both BCGs are probably the result of a tidal trigger. High-sensitivity $\mathrm{HI}$ mapping of the vicinities of other such galaxies would help to better understand the trigger mechanisms of XMD BCG starbursts.

\section{Summary and conclusions}

In this work we present the physical parameters of a new LSB/dIrr galaxy discovered in the vicinity of one of the most metal-deficient BCGs, HS 0822+3542. Its small relative velocity and projected distance are consistent with a scenario of tidal disturbance exerted on the BCG progenitor, which triggered gas collapse and a subsequent SF burst.

The total $(B-V)$ and $(V-R)$ colours of SAO $0822+3545$ are unusually blue in comparison to the most of the known LSBD/dIrr galaxies. This suggests that there was some recent elevated SF in this galaxy. The most straightforward interpretation of the LSBD colours and $E W$ of $\mathrm{H} \alpha$ emission imply that a substantial fraction of its stars are young, with ages from $\sim 10$ to $\sim 1$ Gyr, depending on the model IMF used. Recent deep spectroscopy of HS $0822+3542$ (Izotov et al., in preparation) indicates the existence of a stellar population with the ages of 50-100 Myr. This is comparable to the age of the suggested "intermediate" or "old" stellar populations in the LSBD. Thus, in both components of the pair a substantial fraction of stars formed recently, on a time scale of 100-200 Myr. This suggests that this recent SF was triggered by the mutual tidal action of these dwarfs.

This conclusion on the interaction-induced SF episodes in these dwarfs is a preliminary one, based mainly on some general estimates and the understanding that such an unusual pair of galaxies is unlikely to occur by chance. Another important property of this pair is its high degree of isolation from known bright galaxies. This presumably led to retarded star formation and/or slow chemical evolution in HS $0832+3542$, and a very low SFR in SAO $0822+3545$, as evidenced by their very high gas-mass fractions.

To better understand the evolutionary status of SAO $0822+3545$, one needs higher precision in both integrated colours and those of the outermost parts of the LSBD. This can provide direct confirmation of the composite population model. $\mathrm{H} \alpha$ images will help to determine the spatial distribution of the recent SF episode, while colour-magnitude diagrams for resolved stars will also provide direct age estimates for older stellar populations.

From the results and discussion above we draw the following conclusions:

1. BCG HS $0822+3542$ has a physically associated LSB/dIrr galaxy at a projected distance of $\sim 11.4 \mathrm{kpc}$ with a close radial velocity $\left(\Delta V \lesssim 25 \mathrm{~km} \mathrm{~s}^{-1}\right)$.

2. This LSB/dIrr galaxy, named SAO $0822+3545$, has a low luminosity $\left(\mathrm{M}_{B}^{0}=-12.85\right)$ and small optical size $\left(D_{25} \sim\right.$ $1.5 \mathrm{kpc})$. Its unusually blue integrated colours $(B-V)_{0}=$ 0.08 and $(V-R)_{0}=0.14$, for the case of a standard Salpeter IMF with $M_{\text {up }}=120 M_{\odot}$, indicate recent $(t \sim 10-200 \mathrm{Myr})$ SF episodes, in which stars comprising from $\sim(6-7) \%$ to $\sim 100 \%$ of the total stellar mass, have formed. The total stellar mass comprises $\sim(7-27) \%$ of the galaxy's baryon mass, depending on the age of the "old" stellar component.

3. Alternatively, if its IMF has $M_{\text {up }}=30 M_{\odot}$ and the same slope, the galaxy's properties can be explained by one component continuous SF during the last $\sim 1$ Gyr. This, however, is less probable, considering the expected effects of a recent encounter.

4. New, high-S/N-ratio spectra of HS $0822+3542$ yield an oxygen abundance of $12+\log (\mathrm{O} / \mathrm{H})=7.44$, consistent within the observational uncertainties with earlier measurements. The precision of N/O is improved, and the abundance of argon is derived for the first time.

5. In HS $0822+3542$, for the first time the kinematic evidence has been found of a large ionized-gas supershell, with a diameter of $\sim 0.5 \mathrm{kpc}$. The physical parameters of the supershell imply that the current SF episode in this BCG has lasted at least 5-9 Myr.

6. The dwarf pair is situated deep within the nearby LynxCancer void, and thus has not been tidally disturbed by massive galaxies since its formation epoch. All available data are consistent with the hypothesis that we are witnessing the results of an interaction a few hundred Myr ago between two very gas-rich dwarf galaxies with significantly different masses and density distributions.

Acknowledgements. The authors thank J.Chengalur for permission to cite the GMRT results from a paper in preparation. We are grateful to Y.Izotov and N.Bergvall, who have critically read the early versions of the paper and made many useful suggestions. We are thankful to the anonymous referee, whose criticism and useful suggestions helped to improve significantly the original version of the paper. We acknowledge partial support from INTAS grant 96-0500 and from the Russian state program "Astronomy". This research has made use of the NASA/IPAC Extragalactic Database (NED), which is operated by the Jet Propulsion Laboratory, California Institute of Technology, under contract with the National Aeronautics and Space Administration, and LEDA, the Lyon-Meudon extragalactic database. The use of the Digitized Palomar Observatory Sky Survey (DPOSS-II) and APM Database is gratefully acknowledged. 


\section{References}

Adorf, H. M., Hook, R. N., Lucy, L. B., \& Murtagh, F. D. 1992, in 4th ESO/ST-ECF Data Analysis Workshop, Garhing, ed. P. J. Grosbøl, \& R. C. E. de Ruijsscher, 99

Afanasiev, V. L., Burenkov, A. N., Vlasyuk, V. V., \& Drabek, S. V. 1995, SAO RAS internal report No. 234

Bohlin, R. C. 1996, AJ, 111, 1743

Bothun, G. D., Schombert, J. M., Impey, C. D., Sprayberry, D., \& McGaugh, S. S. 1993, AJ, 106, 530

Burkholder, V., Impey, C., \& Sprayberry, D. 2001, AJ, 122, 2318

Chengalur, J. N., Giovanelli, R., \& Haynes, M. P. 1995, AJ, 109, 2415

Chengalur, J. N., Pustilnik, S. A., Martin, J. -M., \& Kniazev, A. Y. 2003, A\&A, in preparation

Dalcanton, J. J., Spergel, D. N., Gunn, J. E., Smith, M., \& Schneider, D. P. 1997, AJ, 114, 635

Dufour, R., Esteban, C., \& Castañeda, H. 1996, ApJ, 471, L87

de Blok, W. J. G., van der Hulst, J. M., \& Bothun, G. D. 1995, MNRAS, 274, 235

Efremov, Y. N., Pustilnik, S. A., Kniazev, A. Y., et al. 2002, A\&A, 389,855

Fairall, A. 1998, Large-Scale Structures in the Universe, Whiley-Praxis, Chichester, 196 pp.

Ferland, G. J. 1996, NAZY, a brief introduction to CLOUDY, Univ. of Kentucky, Dep. of Physics and Astronomy Internal Report

Fioc, M., \& Rocca-Volmerange, B. 1997, A\&A, 326, 950

Fioc, M., \& Rocca-Volmerange, B. 2000, [astro-ph/9912179]

Fricke, K., Izotov, Y. I., Papaderos, P., Guseva, N. G., \& Thuan, T. X. 2001, AJ, 121, 169

Gerritsen, J. P. E., \& de Blok, W. J. G. 1999, A\&A, 342, 655

Guseva, N. G., Izotov, Y. I., \& Thuan, T. X. 2000, ApJ, 531, 776

Hook, R., \& Lucy, L. B. 1992, ST-ECF Newsletter, 17, 10

Hunter, D. A. 1997, PASP, 109, 937

Hunter, D. A., \& Gallagher, J. S. 1986, PASP, 98, 5

Icke, V. 1985, A\&A, 144, 115

Impey, C., \& Bothun, G. 1997, ARA\&A, 35, 267

Izotov, Y. I., \& Thuan, T. X. 1999, ApJ, 511, 639

Izotov, Y., Thuan, T., \& Lipovetsky, V. 1994, ApJ, 435, 647

Izotov, Y. I., Lipovetsky, V. A., Chaffee, F., et al. 1997, ApJ, 476, 298

Izotov, Y., Chaffee, F., Foltz, C., et al. 1999, ApJ, 527, 757

Izotov, Y., Chaffee, F., Foltz, C., et al. 2001, ApJ, 560, 222

Karachentsev, I. D., \& Makarov, D. I. 2001, Astrofizika, 44, 5

Karachentsev, I. D., Makarov, D. I., \& Huchtmeier, W. 1999, A\&AS, 139, 97

Kniazev, A. Y. 1997, Ph.D. Thesis, Special Astrophysical Observatory, Nizhnij Arkhyz

Kniazev, A. Y., \& Shergin, V. S. 1995, SAO RAS internal report No. 249, 1

Kniazev, A., Pustilnik, S., Masegosa, J., et al. 2000, A\&A, 357, 101

Kniazev, A. Y., Pustilnik, S. A., Pramsky, A. G., \& Ugryumov, A. V. 2001, A\&A, 371, 404

Kunth, D., \& Östlin, G. 2000, A\&ARev, 10, 1

Kunth, D., \& Östlin, G. 2001, in Proc. of Euroconf. Evolution of galaxies, ed. G. Stasinska, E. Perez, \& J. Vilchez, ApSSS, 277, 281

Leitherer, C. 1994, Rev. Mod. Astron., 7, 73, ed. G. Klare (Hamburg: Astronomische Gesellschaft)

Leitherer, C., \& Heckman, T. M. 1995, ApJS, 96, 9

Leitherer, C., Schaerer, D., Goldader, J. D., et al. 1999, ApJS, 123, 3

Lipovetsky, V. A., Chaffee, F., Izotov, Y. I., et al. 1999, ApJ, 519, 177

Mac Low, M. M., \& McCray, R. 1988, ApJ, 324, 776

Makarova, L. N. 1999, A\&AS, 139, 491

Makarova, L. N., \& Karachentsev, I. D. 1998, A\&AS, 133, 181
Makarova, L. N., Karachentsev, I. D., Takalo, L. O., Helnänäki, P., \& Valtonen., M. 1998, A\&AS, 128, 459

Marlowe, A. T., Heckman, T. M., Wyse, R. F. G., \& Schommer, R. 1995, ApJ, 438, 563

Martin, C. 1996, ApJ, 465, 680

Martin, C. 1997, ApJ, 491, 561

Martin, C. 1998, ApJ, 506, 222

McGaugh, S. 1996, MNRAS, 280, 337

McGaugh, S. S., \& Bothun, G. D. 1994, AJ, 107, 530

Mihos, J. C., McGaugh, S. S., \& de Blok, W. J. G. 1997, ApJ, 477, L79

Moy, E., Rocca-Volmerange, B., \& Fioc, M. 2001, A\&A, 365, 347

Noeske, K. G., Iglesias-Páramo, J., Vilchez, J. M., Papaderos, P., \& Fricke, K. J. 2001, A\&A, 371, 806

O’Neil, K., \& Bothun, G. 2000, ApJ, 529, 811

O’Neil, K., Bothun, G., Schombert, J., Cornell, M., \& Impey, C. 1997, AJ, 114, 2448

O’Neil, K., Bothun, G., \& Schombert, J. 1998, AJ, 116, 2776

Östlin, G. 2000, ApJ, 535, L99

Östlin, G., \& Kunth, D. 2001, A\&A, 371, 429

Papaderos, P., Izotov, Y. I., Fricke, K. J., Thuan, T. X., \& Guseva, N. G. 1998, A\&A, 338, 43

Papaderos, P., Izotov, Y. I., Thuan, T. X., et al. 2002, A\&A, 393, 461

Peebles, P. J. E. 2001, ApJ, 557, 495

Pilyugin, L. S. 2001, A\&A, 374, 412

Pustilnik, S. A., Lipovetsky, V. A., Izotov, Y. I., et al. 1997, Astron. Lett., 23, 308

Pustilnik, S. A., Brinks, E., Thuan, T. X., Lipovetsky, V. A., \& Izotov, Y. I. 2001a, AJ, 121, 1413

Pustilnik, S. A., Kniazev, A. Y., Lipovetsky, V. A., \& Ugryumov, A. V. 2001b, A\&A, 373, 24

Pustilnik, S. A., Martin, J. -M., Huchtmeier, W., et al. 2002, A\&A, 389, 405

Ronnback, J., \& Bergvall, N. 1994, A\&AS, 108, 193

Salzer, J. J., di Serego Alighieri, S., Matteucci, F., Giovanelli, R., \& Haynes, M. P. 1991, AJ, 101, 1258

Schlegel, D., Finkbeiner, D., \& Douglas, M. 1998, ApJ, 500, 525

Schombert, J., McGaugh, S., \& Eder, J. 2001, AJ, 121, 2420

Searle, L., \& Sargent, W. L. W. 1972, ApJ, 173, 25

Silich, S. A., Franco, J., Palous̃, J., \& Tenorio-Tagle, G. 1996, ApJ, 468,722

Skillman, E. D., Kennicutt, R. C., \& Hodge, P. W. 1989, ApJ, 347, 875

Taylor, C. L., Brinks, E., Grashuis, R. M., \& Skillman, E. D. 1995, ApJS, 99, 427

Tenorio-Tagle, G. 1996, AJ, 111, 1641

Tenorio-Tagle, G. \& Bodenheimer, P. 1988, ARAA, 26, 145

Thornton, K., Gaudlitz, M., Janka, H. -T., \& Steinmetz, M. 1998, ApJ, 500, 95

Ugryumov, A. V., Engels, D., Pustilnik, S. A., et al. 2003, A\&A, 397, 463

van Zee, L. 2000, ApJ, 543, L31

van Zee, L. 2001, AJ, 121, 2003

van Zee, L., Haynes, M. P., \& Salzer, J. J. 1997, AJ, 114, 2497

van Zee, L., Westpfahl, D., Haynes, M. P., \& Salzer, J. J. 1998, AJ, 115,1000

van Zee, L., Salzer, J. J., \& Skillman, E. D. 2001, AJ, 122, 121

Walter, F., \& Brinks, E. 1999, AJ, 118, 273

Weaver, R., McCray, R., Castor, J., Shapiro, P., \& Moore, R. 1977, ApJ, 218, 377

Whitford, A. E. 1958, AJ, 63, 201

Zasov, A. V., Kniazev, A. Y., Pustilnik, S. A., et al. 2000, A\&AS, 144, 429 